\title{
Live cell analyses of synaptonemal complex dynamics and chromosome movements in cultured mouse testis tubules and embryonic ovaries
}

\author{
Andrea Enguita-Marruedo ${ }^{1}$ - Wiggert A. Van Cappellen ${ }^{2} \cdot$ Jos W. Hoogerbrugge $^{1} \cdot$ Fabrizia Carofiglio $^{1}$. \\ Evelyne Wassenaar ${ }^{1} \cdot$ Johan A. Slotman ${ }^{2}$. Adriaan Houtsmuller ${ }^{2} \cdot$ Willy M. Baarends ${ }^{1}$
}

Received: 23 August 2017 / Revised: 12 March 2018 / Accepted: 12 March 2018 / Published online: 26 March 2018

(C) The Author(s) 2018

\begin{abstract}
During mammalian meiotic prophase, homologous chromosomes connect through the formation of the synaptonemal complex (SC). SYCP3 is a component of the lateral elements of the SC. We have generated transgenic mice expressing N- or C-terminal fluorescent-tagged SYCP3 (mCherry-SYCP3 (CSYCP) and SYCP3-mCherry (SYCPC)) to study SC dynamics and chromosome movements in vivo. Neither transgene rescued meiotic aberrations in Sycp 3 knockouts, but CSYCP could form short axial element-like structures in the absence of endogenous SYCP3. On the wild-type background, both fusion proteins localized to the axes of the SC together with endogenous SYCP3, albeit with delayed initiation (from pachytene) in spermatocytes. Around $40 \%$ of CSYCP and SYCPC that accumulated on the SC was rapidly exchanging with other tagged proteins, as analyzed by fluorescent recovery after photobleaching (FRAP) assay. We used the CSYCP transgenic mice for further live cell analyses and observed synchronized bouquet configurations in living cysts of two or three zygotene oocyte nuclei expressing CSYCP, which presented cycles of telomere clustering and dissolution. Rapid chromosome movements were observed in both zygotene oocytes and pachytene spermatocytes, but rotational movements of the nucleus were more clear in oocytes. In diplotene spermatocytes, desynapsis was found to proceed in a discontinuous manner, whereby even brief chromosome re-association events were observed. Thus, this live imaging approach can be used to follow changes in the dynamic behavior of the nucleus and chromatin, in normal mice and different infertile mouse models.
\end{abstract}

Keywords Meiotic prophase $\cdot \mathrm{SYCP} 3 \cdot$ in vitro culture $\cdot$ Spermatocyte $\cdot$ Oocyte $\cdot$ Synaptonemal complex

\section{Introduction}

In all sexually reproducing diploid species, homologous chromosomes must separate faithfully during the first meiotic division, to generate two haploid cells. Leading up to this event, homologs must form pairs. This requires dynamic movements of chromosomes inside the nucleus, as has been visualized in living rodent spermatocytes using transillumination

Electronic supplementary material The online version of this article (https://doi.org/10.1007/s00412-018-0668-7) contains supplementary material, which is available to authorized users.

Willy M. Baarends

w.baarends@erasmusmc.nl

1 Department of Developmental Biology, Erasmus MC University Medical Centre, Rotterdam, The Netherlands

2 Department of Pathology, Erasmus Optical Imaging Centre, Erasmus MC University Medical Centre, Rotterdam, The Netherlands microscopy already several decades ago (Parvinen and Soderstrom 1976; Salonen et al. 1982). One of the most conspicuous features of meiotic prophase cells that has been observed in almost all analyzed sexually reproducing species to date is the so-called bouquet stage, when telomeres cluster together in the nuclear periphery, and the thread-like chromosomes form a structure that is reminiscent of a bouquet of flowers (Scherthan 2007; Stewart and Burke 2014). In addition, rapid telomere movements occur in both yeast and mouse, and both processes require linkage of the telomeres to the cytoskeleton, through the nuclear membrane (Lee et al. 2012; Lee et al. 2015). These dynamic processes occur handin-hand with the initiation of homologous chromosome pairing. Correct chromosome pairing also requires the formation and repair of DNA double-strand breaks, mediated by the SPO11/TOPOVIBL complex (Baudat et al. 2000; Robert et al. 2016; Romanienko and Camerini-Otero 2000), together with additional meiosis-specific proteins. In addition, a DSBindependent role of SPO11 in early homologous chromosome 
interactions has been described (Boateng et al. 2013). Pairing results in synapsis, defined as the formation of a physical proteinaceous connection between the chromosomes, in a zipper-like fashion along the chromosomal arms. This connecting protein structure is called the synaptonemal complex (SC) and is composed of two lateral elements (LEs, one per homolog) that associate with each other through the transverse filaments (TFs). They overlap in the central region, forming the central element (CE) (Page and Hawley 2004). In mammals, the LEs and their precursors, the axial elements (AEs), are mainly composed of the proteins SYCP2 and SYCP3, while the TFs mainly consist of SYCP1 (Costa et al. 2005; Heyting 1996). The CE contains SYCP1 as well as other proteins like SYCE1, SYCE2, SYCE3, and TEX1 (Costa et al. 2005; Hamer et al. 2006; Schramm et al. 2011).

With the advent of technologies to express fluorescenttagged proteins, and the means to analyze their expression in living cells using confocal fluorescent microscopy, more possibilities to investigate chromosome movement in detail have arisen. In mice carrying a SYCP3-EYFP transgene driven by a $P g k 2$-promoter, labeled SCs were analyzed in cultured pachytene spermatocytes embedded in a fibrinogen clot for periods of up to $6 \mathrm{~h}$ (Morelli et al. 2008). In these nuclei, only subtle movements were detected. More recently (Shibuya et al. 2014) used in vivo DNA electroporation to express fluorescent-tagged SYCP3 and TRF1 in mouse spermatocytes. Cultured cells were analyzed for periods no longer than $10 \mathrm{~min}$. The analysis of the SYCP3 behavior showed rapid movements of chromosomes within the nuclei throughout meiotic prophase. A recent study (Rog and Dernburg 2015) developed in the model organism C. elegans, involved analyses of the behavior of the $\mathrm{SC}$ in the worms in vivo.

We aimed to study the dynamics of chromosomes and the $\mathrm{SC}$ in living mouse spermatocytes or oocytes, in their natural environment (inside the seminiferous tubule or the ovary). We have generated transgenic mice expressing $\mathrm{N}$ - or $\mathrm{C}$-terminal fluorescent-tagged SYCP3-mCherry, and developed a method that allows us to culture seminiferous tubules or ovaries during short-term (minutes) and long-term overnight cellimaging experiments. SYCP3 is an important functional component of the axial/lateral elements of the SC. Disruption of Sycp3 leads to aberrant chromosome pairing and synapsis, and spermatocytes do not progress further than a zygotene-like stage (Hamer et al. 2008; Royo et al. 2010). Female Sycp $3^{-1-}$ knockouts show milder defects, and they are subfertile, showing a reduction in litter size (Yuan et al. 2002).

Here, we show that both $\mathrm{N}$-terminal and $\mathrm{C}$-terminal tagging of SYCP3 precludes formation of functional SYCP3 filaments in the absence of endogenous, untagged SYCP3. However, when untagged SYCP3 is also expressed, the tagged proteins can accumulate on the axial elements, exchange dynamically, and do not interfere with normal progression of oogenesis and spermatogenesis. This allowed us to perform detailed analyses of chromosome and nuclear movements during early and late meiotic prophase in oocytes and spermatocytes, respectively. Thereby, this study provides novel insight in nuclear rotation speed, dynamics of bouquet formation, and progression of desynapsis in living mouse meiocytes in a tissue context.

\section{Results}

\section{Transgenic expression of fluorescent-tagged SYCP3}

To drive expression of the transgenes encoding SYCP3 tagged with mCherry at either the $\mathrm{N}$-or $\mathrm{C}$-terminal end of the protein, we used a promoter fragment of $S m c 1 b$, previously reported to drive specific expression in spermatocytes from leptotene onwards (Fig. S1a) (Adelfalk et al. 2009). We confirmed expression of the tagged protein first on Western blot, using total testis protein extracts from mice of different age. A single band was observed for N-terminally tagged SYCP3 (CSYCP), but C-terminal-tagged SYCP3 (SYCPC) was expressed in two forms, most likely as a result of the presence of two possible start codons in the first exon, as previously reported (Alsheimer et al. 2010). The levels of the two tagged proteins were lower compared to the level of endogenous SYCP3, and expression initiated later during postnatal development (Fig. S1b). Thus, although endogenous SMC1B and SYCP3 are known to display very similar expression patterns in spermatocytes (Dobson et al. 1994; Revenkova et al. 2001), the $S m c 1 b$ promoter fragment used here resulted in reduced and delayed expression of fluorescent-tagged SYCP3 compared to endogenous SYCP3.

\section{SYCPC and CSYCP locate on the axis of the fully formed SC in spermatocytes, and also on axial elements in oocytes on a wild-type background}

Next, we analyzed the localization of both fusion proteins in nuclear spread preparations of spermatocytes and oocytes, whereby the transgene was expressed either on a wild-type or Sycp $3^{+/}$background. We detected both endogenous and transgenic SYCP3, making use of anti-SYCP3 antibody, and could selectively analyze the tagged protein by detecting the fluorescent signal from the mCherry moiety.

In males, SYCPC as well as CSYPC were detected from pachytene onwards, along the lateral elements of the SC, colocalizing with endogenous SYCP3 (Fig. 1). Interestingly, both fusion proteins displayed a signal of higher intensity at telomeric ends of the SC of the autosomes (Fig. 1a, b, enlargements in mid pachytene) and also along the axial elements of the $\mathrm{X}$ and $\mathrm{Y}$ chromosomes (Fig. 1a, b, mCherry signal in mid pachytene). Enrichment of fusion protein on the XY pair was most evident for CSYCP (Fig. 1b, mCherry signal in mid 
Fig. 1 Expression of SYCPC and CSYCP in wild-type spermatocytes. Immunostaining of SYCP3 (green) on Sycp $3^{+/}$SYCPC (a) and $\operatorname{Sycp} 3^{+-}$CSYCP (b) spermatocyte nuclei at different stages of meiotic prophase. The red signal represents the mCherry signal (no antibody detection). Scale bar $10 \mu \mathrm{m}$ a

\section{Spermatocytes}
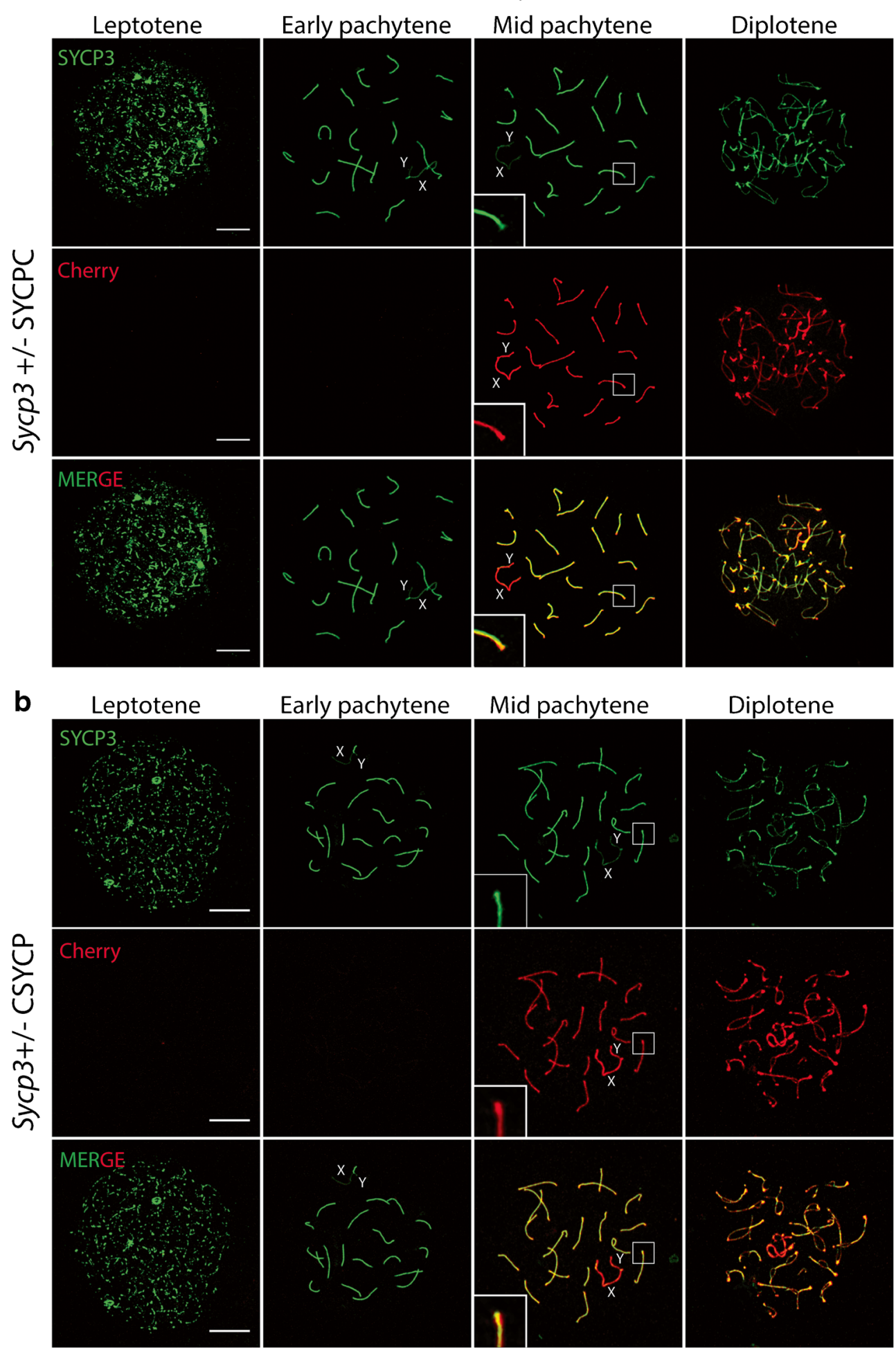

pachytene). In contrast, the overall SYCP3 signal (Fig. 1a, b, SYCP3 in mid pachytene, consisting of endogenous as well as tagged protein) was lower along the non-synapsed $\mathrm{XY}$ axes, compared to the synapsed autosomes, and no thickening was observed at the SC ends.
In embryonic ovaries, CSYCP and SYCPC were detected in oocyte nuclei from leptotene (embryonic day 16.5 (E16.5) until diplotene (E18.5) stages (Fig. 2a, b, staging of oocytes is described in the "Materials and methods" section). Also here, the tagged proteins colocalized with endogenous SYCP3. We 
Fig. 2 Expression of SYCPC and CSYCP in wild-type oocytes. Immunostaining of SYCP3 (green) and RAD51 (white, pseudo-color from infrared) on Sycp $3^{+/+}$SYCPC (a) and Sycp $3^{+/}$CSYCP (b) oocyte nuclei from embryonic ovaries isolated at E16.5 (early and late zygotene and pachytene) and E18.5 (diplotene). mCherry (red) is visualized directly (no antibody detection). Scale bar $10 \mu \mathrm{m}$ a

\section{Oocytes}

early zygotene late zygotene pachytene diplotene

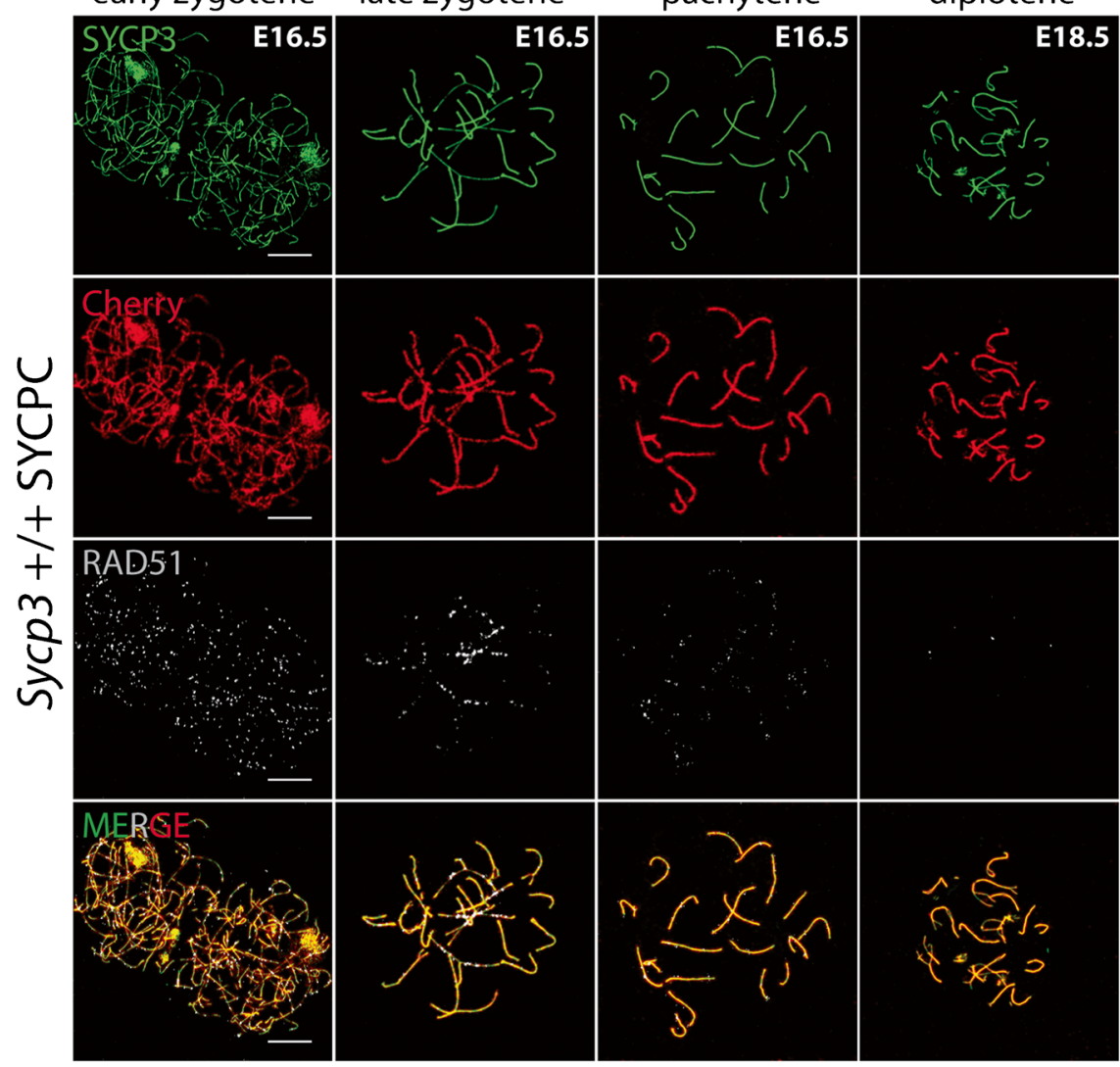

b early zygotene late zygotene pachytene diplotene

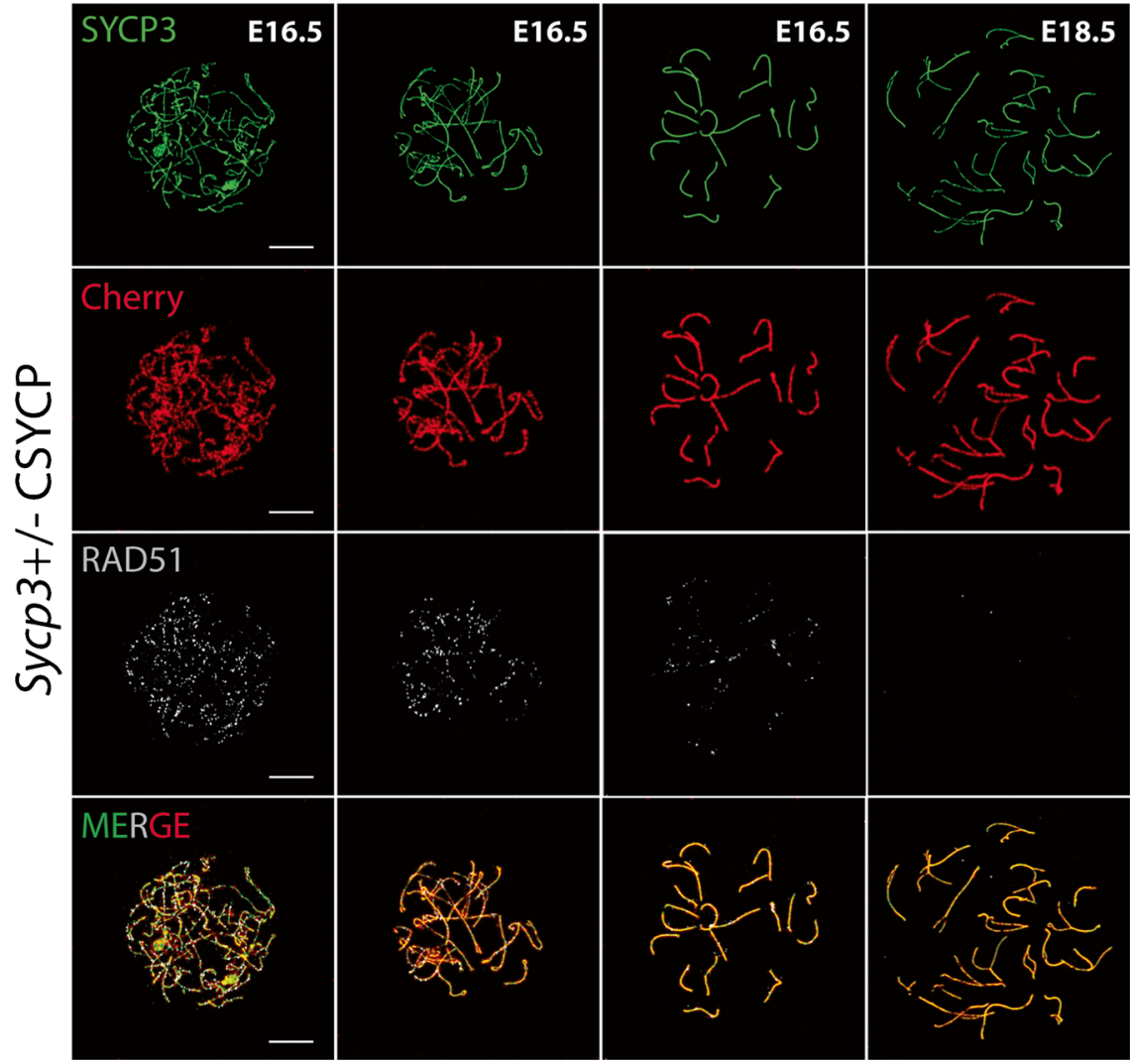


did not detect either fusion protein in ovaries isolated from embryos younger than E16.5. No differences were observed between the patterns of the two fusion proteins, and also not between the overall SYCP3 signal and that of the mCherrytagged protein only. The fact that both SYCPC and CSYCP localize to axial elements in leptotene and zygotene oocytes, but not in such early spermatocyte nuclei, is most likely due to the fact that the $S m c 1 b$ promoter fragment used in our transgene constructs is not yet active in leptotene and zygotene spermatocytes in our models (see the "Discussion" section).

\section{mCherry-tagged SYCP3 does not interfere with normal meiotic progression but cannot rescue the Sycp3 knockout phenotype}

Tagging a protein in vivo may interfere with its normal function. In addition, regulation of expression of our transgene differs from that of endogenous SYCP3, which may also influence its degree of functionality. To assess whether the expression of the two SYCP3 fusion proteins interfered with normal progression of meiosis, we assessed overall fertility (Fig. S2a), crossover frequency (Fig. S2b), and persistence of DSBs in late meiotic prophase (Fig. S2c) in males carrying the transgene in addition to one or two wild-type Sycp3 alleles. None of these parameters were affected. To investigate to what extent the SYCPC and CSYCP proteins were capable of functionally replacing endogenous SYCP3, we analyzed the meiotic phenotype of Sycp3 $3^{-/-}$male and female mice, in the presence or absence of SYCPC and CSYCP. Prophase substages were determined using the known stage-specific localization pattern of the meiosis-specific cohesin component REC8, which mostly colocalizes with SYCP3 in wild-type spermatocytes and oocytes (Lee et al. 2003). In male Sycp3 $3^{-/}, S_{y c p} 3^{-/}$SYCPC, and Sycp $3^{-/}$CSYCP spreads, we observed leptotene and zygotene-like stages (Fig. 3a-c), but no cells in which complete synapsis was achieved. In addition, no SYCPC protein was detected in Sycp $3^{-/}$SYCPC males, upon staining with anti-SYCP3 (Fig. 3b). In contrast, CSYCP could be detected as small patches along the axial elements (Fig. 3c) in $67 \%$ of the nuclei analyzed ( $n=52$ nuclei, 2 mice). When CSYCP was present, it mostly colocalized with REC8 (Fig. 3c, enlargements). Immunodetection of SYCP1 (marker of synapsis) revealed that the degree of synapsis in $\mathrm{Sycp}^{-/-}$CSYCP, Sycp $3^{-/}$, and Sycp $3^{-/-}$SYCPC spermatocytes was similar (Fig. 3d-f). Interestingly, CSYCP accumulation was less apparent on (heterologously) synapsed SC fragments compared to unsynapsed axial elements (Fig. 3f, enlargements). To further evaluate functionality of SYCPC and CSYCP, we analyzed if our tagged SYCP3 proteins would alter the pattern of SYCP2 accumulation on the knockout background, since it has been reported that SYCP2 fails to localize to the axes upon knockout of Sycp3 (Pelttari et al. 2001), and vice versa (Yang et al. 2006). However, in contrast to the published results, we observed that $\mathrm{SYCP} 2$ was still present on the axes of $S y c p 3^{-1-}$ spermatocytes, and also of $S y c p 3^{-1-}$ SYCPC and Sycp3 ${ }^{-/}$CSYCP spermatocytes (Fig. S3a,b). Identical results were obtained with two different antibodies targeting SYCP2. Thus, it appears that SYCP2 localization does not depend on SYCP3 expression and is not influenced by the presence of mCherry-tagged SYCP3. Together, the data indicate that the $S y c 3^{-/}$phenotype is not rescued by either transgene in males.

We then determined if SYCPC and CSYCP proteins could functionally replace SYCP3 in females. Similar to what was observed in males, SYCPC did not localize to axial elements in the absence of endogenous SYCP3 in E16.5 leptotene and zygotene oocytes (Fig. S4a) but showed focal accumulation on chromatin (not in association with axial elements) at later stages, in late pachytene/diplotene-like oocytes at E18.5 (Fig. 4b). CSYCP already accumulated on the chromatin at leptotene and zygotene in E16.5 Sycp $3^{-/}$CSYCP oocytes (Fig. S4b) and clearly displayed axial localization when cells reached a pachytene-like stage (E16.5, Fig. 4a and S4b; E18.5, Fig. 4b). However, the protein covered only part of the axes visualized by anti-REC8. During normal meiotic prophase progression, RAD51 foci numbers (indicative of DSB repair sites) gradually decrease and disappear in diplotene. In contrast, RAD51 foci are retained in $\mathrm{Sycp3}^{-/-}$pachytene and diplotene oocytes (Wang and Hoog 2006). The presence of SYCPC or CSYCP did not reduce the number of persisting RAD51 foci in $S y c p 3^{-/}$pachytene and diplotene oocytes at E18.5 (Fig. S5a-c). In addition, MLH1 foci numbers (indicative of crossover sites (Kolas and Cohen 2004; Moens et al. 2002)) did not differ between the genotypes (Fig. 4b). Thus, the tagged proteins do not rescue the $S y c p 3^{-/}$oocyte phenotype either.

\section{Identification of prophase substages in living spermatocytes and oocytes expressing CSYCP}

We setup a method to allow time-lapse analyses of oocytes and spermatocytes in cultured embryonic ovaries and tubule fragments, respectively. We used females (E16.5) and males carrying the CSYCP transgene on the wild-type background. The organs or tubule fragments were cultured in a gelatinous protein mixture (basement membrane extract, BME) to maintain three-dimensional structures (see the "Material and methods" section for more details). Stages from leptotene to pachytene were observed in oocytes (Fig. 5a, upper panel) and identified as follows: leptotene nuclei displayed thin axes, and their telomeres were dispersed in the nucleus (Fig. 5a, upper panel, leptotene). Clustering of telomeres in the periphery of the nucleus was observed during the bouquet stage (Fig. 5a, 
upper panel, zygotene/bouquet). Subsequently, the thickness of the axes varied upon synapsis progression. In pachytene oocytes, all the axes were synapsed (Fig. 5a, upper panel, early pachytene). In the same way, completely synapsed axes were observed in pachytene spermatocytes in $S y c p 3^{+/-}$ CSYCP males (Fig. 5a, lower panel). In late pachytene, thicker chromosome ends and brighter XY axes were observed (Fig. 5a, lower panel, late pachytene). Finally, partially desynapsed axes were observed in diplotene spermatocytes (Fig. 5a, lower panel, diplotene). Although we mainly focused on CSYCP for our time-lapse experiments, similar in vivo observations were made in cultured ovaries and testis tubules from mice expressing SYCPC (see for example Fig. 5b, SYCPC and CSYCP patterns in spermatocytes (imaged at somewhat lower resolution compared to Fig. 5a)).

\section{Synaptonemal complex movements are rapid during prophase}

Movements were highly dynamic during the entire meiotic prophase, in both male and female meiocytes. Two types of movements were observed: rotation of the nucleus itself and movement of the SC inside the nucleus. The rapid movements prompted us to perform an experiment whereby we recorded a single plane with a time lapse of only 1.5-2 $\mathrm{s}$ (this short time frame in between recordings precluded imaging multiple planes). To estimate nuclear rotation speed, we then selected single zygotene oocyte and pachytene spermatocyte nuclei that could be traced in time and calculated rotation angles between two consecutive time points as described in the "Materials and methods" section. We then plotted the cumulative rotation angle against time, whereby rotations in clockwise direction were considered as positive angles and counterclockwise rotations as negative angles (Fig. 5c). Interestingly, oocytes rotated for long time periods in a single direction, whereas spermatocytes presented a kind of "wiggling" movement. However, the oocytes displayed more variability in rotational speed frequency distribution (Fig. 5d), in contrast to the spermatocytes, for which the majority of measured rotation speeds were relatively small $(71 \%$ of speeds between 0.5 and 1.5 degrees/s).

The extensive rotation of the nucleus itself, together with the SC movements, increased complexity to such an extent that we could not follow movement of individual autosomal $\mathrm{SCs}$ in oocytes and spermatocytes. The XY axis in pachytene and diplotene spermatocytes was more easily traceable because of its high mCherry signal. Taking the center of the $\mathrm{XY}$ axis as reference, we found that it moved with a speed of $24 \pm 15 \mathrm{~nm} / \mathrm{s}$ ( $n=10$ nuclei). This displacement is the combined result of nuclear rotation and independent movement of the $\mathrm{XY}$ within the nucleus.
Fig. 3 Expression of SYCPC and CSYCP in Sycp $3^{-/}$spermatocytes. Immunostaining of REC8 (green) and SYCP3 (red) (a-c) or SYCP1 (green) and SYCP3 (red) (d-f) on Sycp $3^{-/}$(a, d), Sycp $3^{-/-}$SYCPC (b,

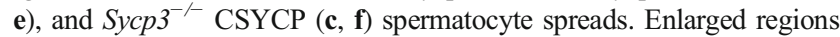
(indicated by white boxes) are shown on the right $(\mathbf{a}-\mathbf{c}, \mathbf{f})$. Below the enlargements in $\mathbf{c}$ and $\mathbf{f}$, a schematic drawing clarifies the merged image. Scale bar $10 \mu \mathrm{m}$

\section{Meiotic prophase progression, including bouquet formation, is synchronized between oocytes in the same cyst}

The fast movements of chromatin and nuclei in early prophase oocytes precluded detailed analyses of the progression of synapsis of individual chromosomes in time. Nevertheless, we could study some other general features in these oocytes. Frequently, we observed two, or sometimes even three, oocytes that moved together as a single unit during the whole recording (from several minutes until a maximum of $10 \mathrm{~h}$ in the overnight experiments). The two or three connected oocytes were always found in the same meiotic stage, indicating synchronized progression through meiosis (videos 1, 2 and 3). The telomeres could be distinguished because of their higher fluorescent signal compared to the rest of the axes, allowing us to observe events of clustering/dissolution of telomeres during the zygotene stage. Clustering and dissolution events sometimes followed each other in rapid succession, even within a few seconds of each other (video 4, bouquet formation can be clearly observed at $t=6 \mathrm{~s}$ in the upper nucleus. Dissolution occurs immediately thereafter). These brief clustering events could be unstable or random events, and we expected that a real functional bouquet event would be maintained for a longer time period. For this reason, we also analyzed bouquet progression in our overnight experiments. In order to prevent problems of bleaching during the long experiment, but to be able to follow nuclei despite the high movement frequency, an interval of $10 \mathrm{~min}$ was chosen for these time-lapse 3D recordings. The fast movements of the chromosomes and nuclei, together with the low resolution in $\mathrm{Z}$, prevented us from obtaining a high-resolution 3D image of the stacks. Still, we could identify bouquet stages using two criteria: clear linear organization of several telomeres next to each other in one of the planes of the z-stack and the continuation of this situation during at least two consecutive time points. This second criterion was also applied to the nonbouquet situation. The observations on different groups of nuclei $(\mathrm{N})(\mathrm{N} 1$ and $\mathrm{N} 4$, experiment 1; N2, N3 and $\mathrm{N} 5$, experiment 2) are summarized in Fig. 6. The nuclei were ordered according to their degree of meiotic progression, whereby $\mathrm{N} 1$ and $\mathrm{N} 2$ were at the earliest stages of prophase, followed by N3, N4, and N5, which represented more and more advanced stages of zygotene development. 

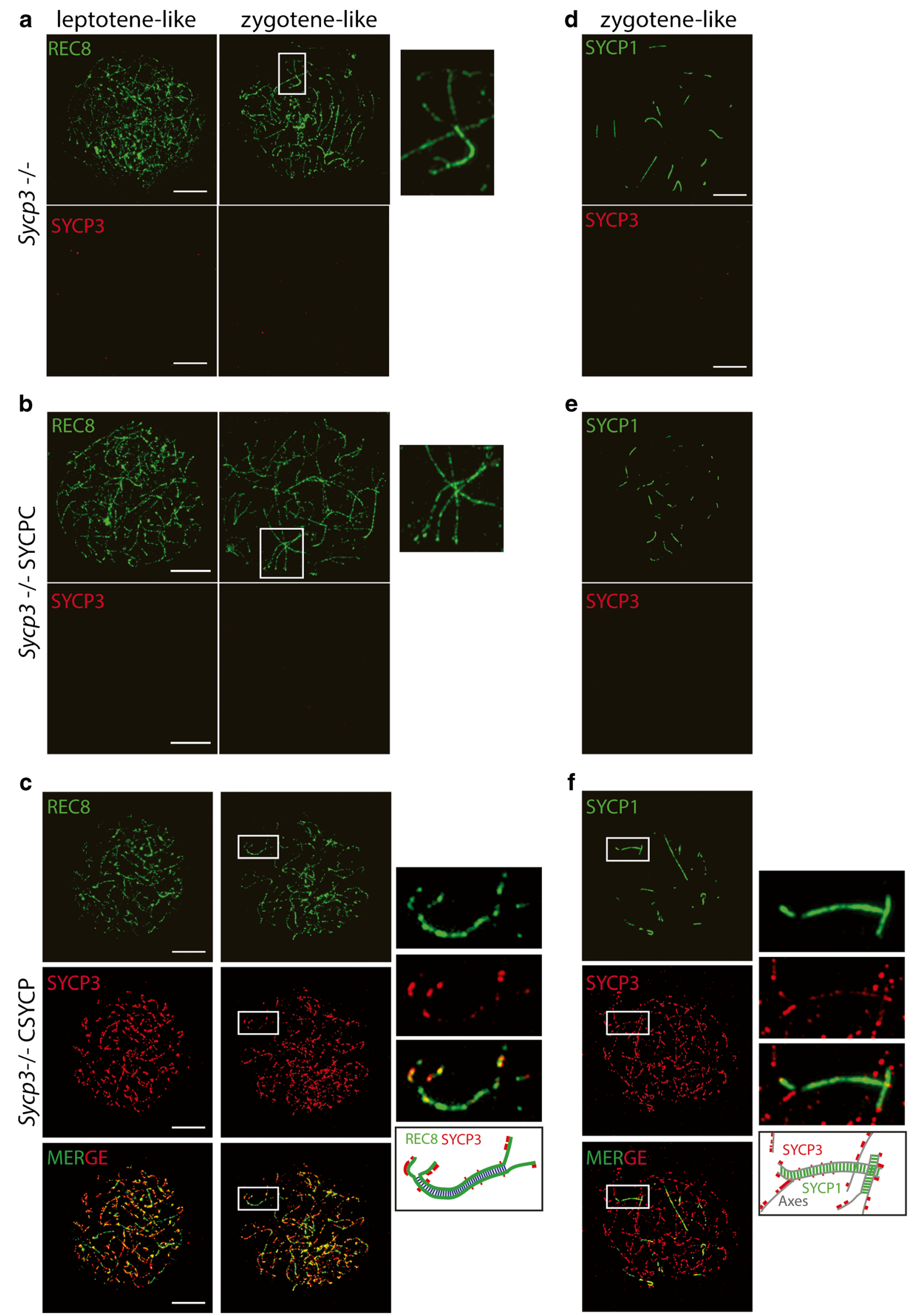
The duration of bouquet configuration varied among these nuclei, ranging from $20 \mathrm{~min}$ (by definition the shortest duration possible) to $2 \mathrm{~h}$ and $10 \mathrm{~min}$. The behavior of the nuclei within one cyst also varied. In $\mathrm{N} 2$ and N5, we observed bouquet formation in only one of the two nuclei. In $\mathrm{N} 1$, one bouquet is dissolved earlier than the other, but in N3 and N4, the nuclei in the cyst behaved synchronously and the bouquet configuration appeared and disappeared approximately at the same time. Some cysts (nucleus B of N3, and nucleus B of N4) exhibited two cycles of telomere clustering, dissolution, and reclustering. Single time point observations of dissolution or clustering could also frequently be observed. As an example, the complete sequence of images for N3 is shown in Fig. S6. Interestingly, when bouquets of two nuclei were present in the same syncytium, they always appeared as if facing each other.

\section{Axis separation starts in interstitial regions and progresses towards the telomeres of the chromosomes}

In diplotene spermatocytes, it was possible to follow the process of axis separation (further referred to as desynapsis) for single chromosome pairs in five bivalents. Desynapsis initiated in the interstitial region of the chromosomes and progressed towards the telomeres (Fig. 7a-e, videos 5-9, representing bivalent a-e, respectively). In the bivalent shown in Fig. 7a and video 5, desynapsis progressed from the desynapsis initiation point indicated with a green arrowhead, while there was no progression of desynapsis from the area indicated with the red arrowhead, indicating that desynapsis does not proceed in a similar fashion from each initiation point on a single bivalent. In all nuclei, desynapsis was discontinuous and even resynapsis was observed for two bivalents (Fig. 7f, nucleus b and e, videos 6 and 9). This can also be inferred from the desynapsis length plotted against time for each individual bivalent that was traced (Fig. 7f). In addition, the frequency distribution of the measured speeds visualizes the variability of the velocity (Fig. 7g: desynapsis (positive values) and resynapsis (negative values)). Around $60 \%$ of the measured velocities were included in the interval between -0.5 and $0.5 \mu \mathrm{m} / \mathrm{min}$, indicating that most of the time period during which the measurements took place was taken up by only minute changes in the degree of axis separation. When visible desynapsis/ resynapsis took place, the most frequently measured speed was around $1 \mu \mathrm{m} / \mathrm{min}$ for both situations (11 and 13\%, respectively). Faster velocities were rare during resynapsis, but relatively frequent during desynapsis $(16 \%$ of the values were between 1.5 and $3.5 \mu \mathrm{m} / \mathrm{min}$ ). In addition, desynapsis events occurred more frequently $(27 \%$ of the measured
Fig. 4 Expression of SYCPC and CSYCP in Sycp $3^{-1-}$ oocytes. a Immunostaining of REC8 (green) and SYCP3 (red) on Sycp $3^{-/-}$ SYCPC and Sycp $3^{-/-}$CSYCP pachytene oocyte nuclei at E16.5. b Immunostaining of REC8 (green), SYCP3 (red), and MLH1 (white, pseudo-color from infrared) on $S y c p 3^{-/-}, S y c p 3^{-1-}$ SYCPC, and Sycp $3^{--}$CSYCP oocyte nuclei at E18.5. Mean of MLH1 foci \pm SD are displayed in the images, $n=7$ nuclei for Sycp $3^{-/-}$and Sycp $3^{-/-}$CSYCP, $n=6$ nuclei for Sycp $^{--}$SYCPC

speeds) than resynapsis (16\%), explaining the overall increase in desynapsis over time for all analyzed bivalents.

\section{CSYCP and SYCPC can be recruited to the lateral elements of the SC}

To determine if CSYCP and SYCPC were stably bound to the lateral elements in spermatocytes, fluorescence recovery after photobleaching (FRAP) was performed. The recovery of at least 10 individual spermatocytes was analyzed per transgenic mouse, expressing either SYCPC or CSYCP in the presence of untagged protein. A single strip that spanned the middle part of the nucleus was photobleached. To reduce technical artifacts due to the high frequency of nucleus and chromosome movement, the nucleus was imaged with a small interval of $0.020 \mathrm{~s}$, and a total time of $1 \mathrm{~min}$, and the XY was never included inside the bleached strip. The average and normalized fluorescence recovery of CSYCP and SYCPC was plotted against time (Fig. 8). A final recovery of $40 \%$ was observed within $1 \mathrm{~min}$ for both CSYCP and SYCPC in the two experiments.

\section{Discussion}

\section{Both $\mathrm{N}$ - and C-terminal fusion of mCherry to SYCP3 affects filament formation}

Neither transgene could rescue the aberrant chromosome pairing and frequent asynapsis that is observed upon knockout of Sycp 3 in the male mice (Yuan et al. 2000). However, while no SYCPC was detected on the axes of spermatocytes and oocytes on a Sycp 3 knockout background, CSYCP could form small patches. Recent results from in vitro analyses have indicated that SYCP3 may assemble in tetramers (Syrjanen et al. 2014). Self-interactions among these tetramers (self-assembly), depending on the last six amino acids of the C-terminal of SYCP3, mediate SYCP3 filament formation in vitro. In these in vitro experiments, the $\mathrm{N}$-terminus appeared to be more important for DNA binding (Syrjanen et al. 2014). We assume that tagging the C-terminus of SYCP3 (SYCPC) precludes de novo assembly of the protein on chromosomal axes but still allows it to associate with DNA at random sites in the chromatin, in the absence of endogenous SYCP3. On the other hand, the intact C-terminus of CSYCP allows it to properly 


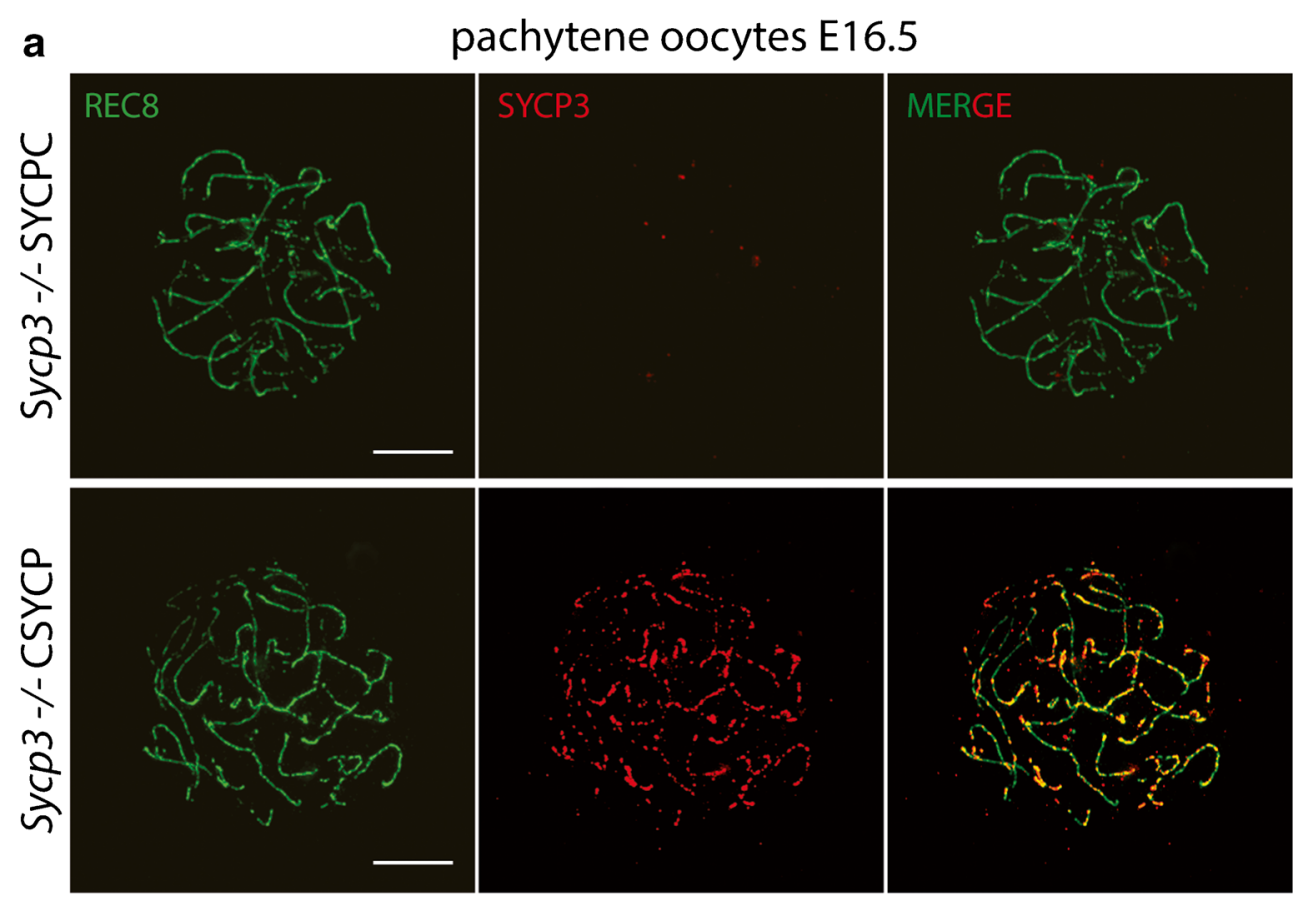

b

late pachytene/diplotene oocytes E18.5

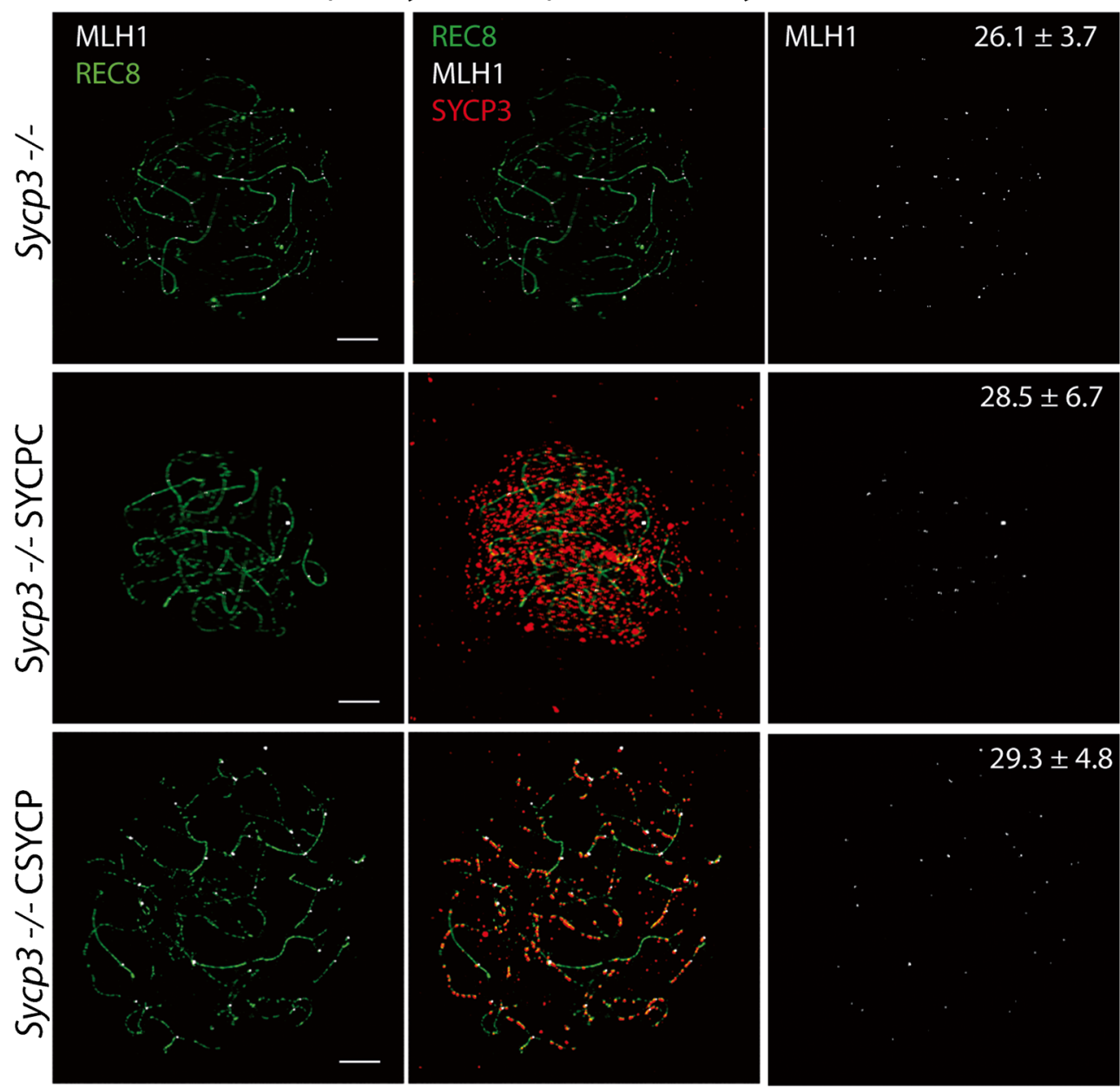


a

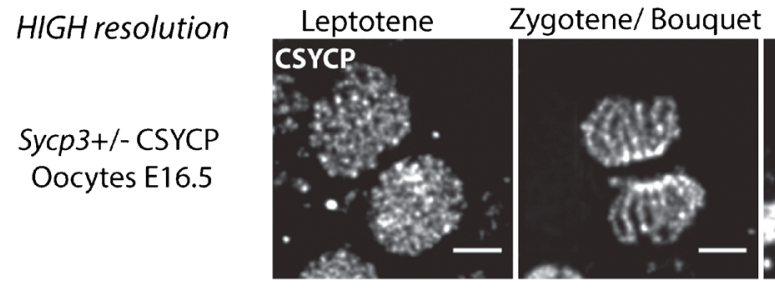

Sycp $3+/-$ CSYCP

No expression

Spermatocytes

\section{b}

LOW resolution

Sycp $3+1-$ CSYCP Spermatocytes

Syсp3+/- SYCPC

No expression

C

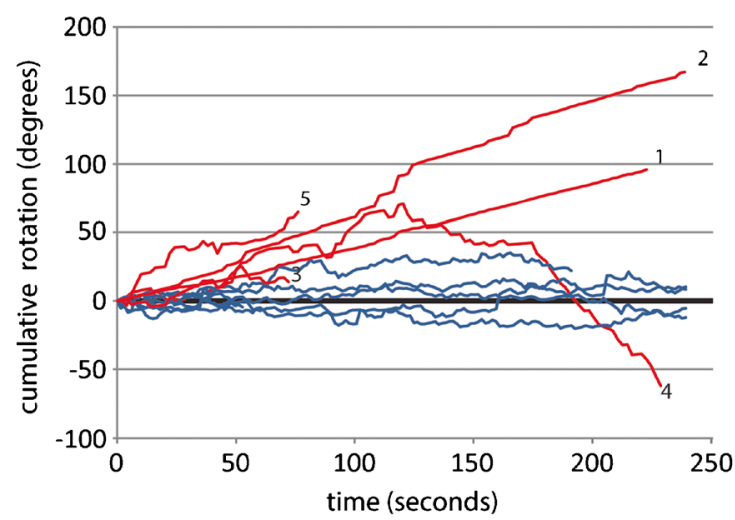

Fig. 5 Live imaging: CSYCP in oocytes and spermatocytes (a) and CSYCP and SYCPC in spermatocytes (b). High-resolution images are depicted in a (obtained using the SP5 confocal microscope, except for the diplotene spermatocyte (lower panel), which was generated using the Airy-scan detector). Green arrowheads in the diplotene spermatocyte indicate desynapsed regions. Low-resolution images are shown in b (SP5 confocal microscope). The low-resolution images display more background and less clear axes. No expression indicates absence of fusion

localize to the axes where oligomers can assemble with each other. However, because the N-terminal tag interferes with higher order structures and/or because the DNA-binding
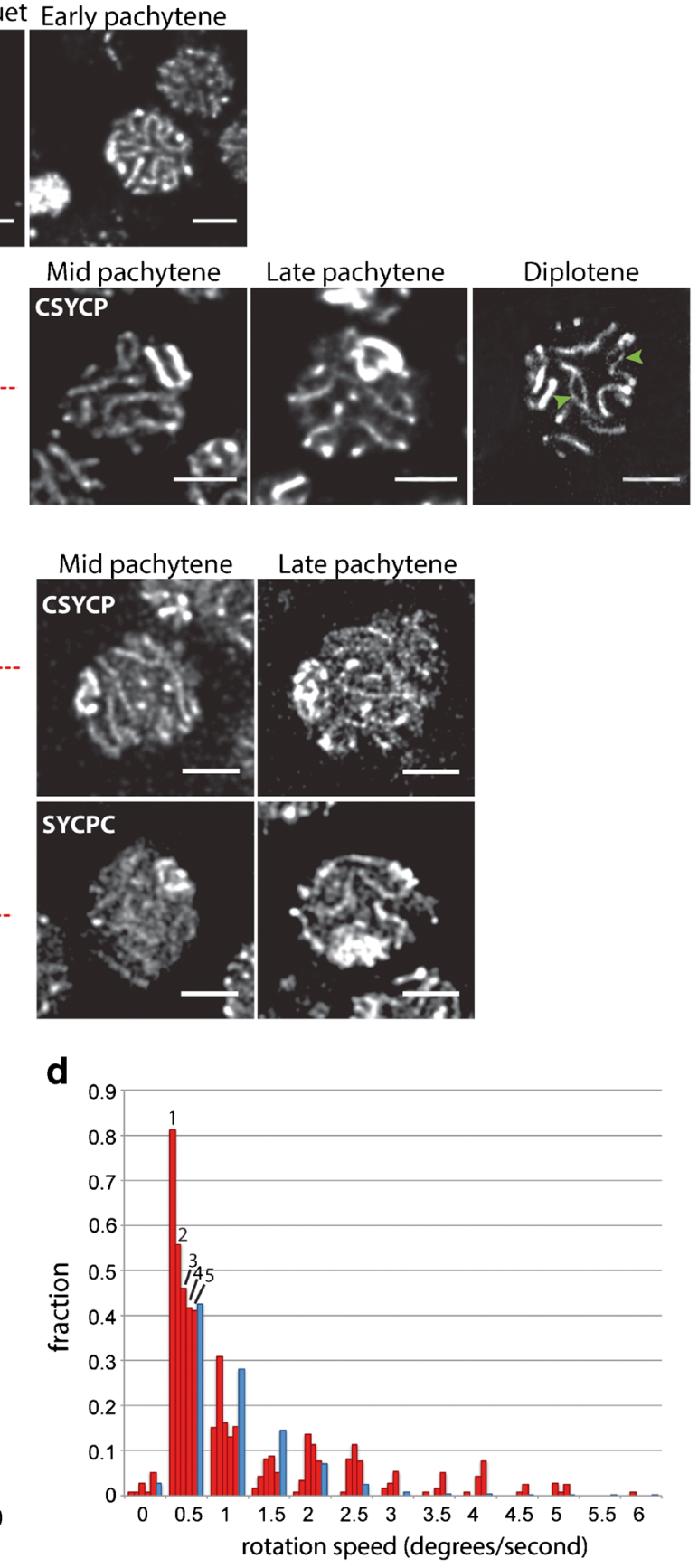

protein expression during leptotene and zygotene stages in spermatocytes. Scale bar $5 \mu \mathrm{m}$. c Cumulative rotations of oocytes (red) and spermatocytes (blue) plotted against time (seconds). Clockwise rotations are added as positive values (Y axis), while counterclockwise rotations are negative values. $\mathbf{d}$ Relative frequencies of rotation speeds of each oocyte (red) and all analyzed spermatocytes (all speeds included, distributions of individual spermatocytes were similar, blue). Same oocyte in $\mathbf{c}$ and $\mathbf{d}$ is indicated with the same number. $N=5$ oocytes and 6 spermatocytes

capacity is affected, only small filaments can be formed. Although we cannot exclude that the added mCherry tags affect overall protein folding, we think that this is unlikely, 

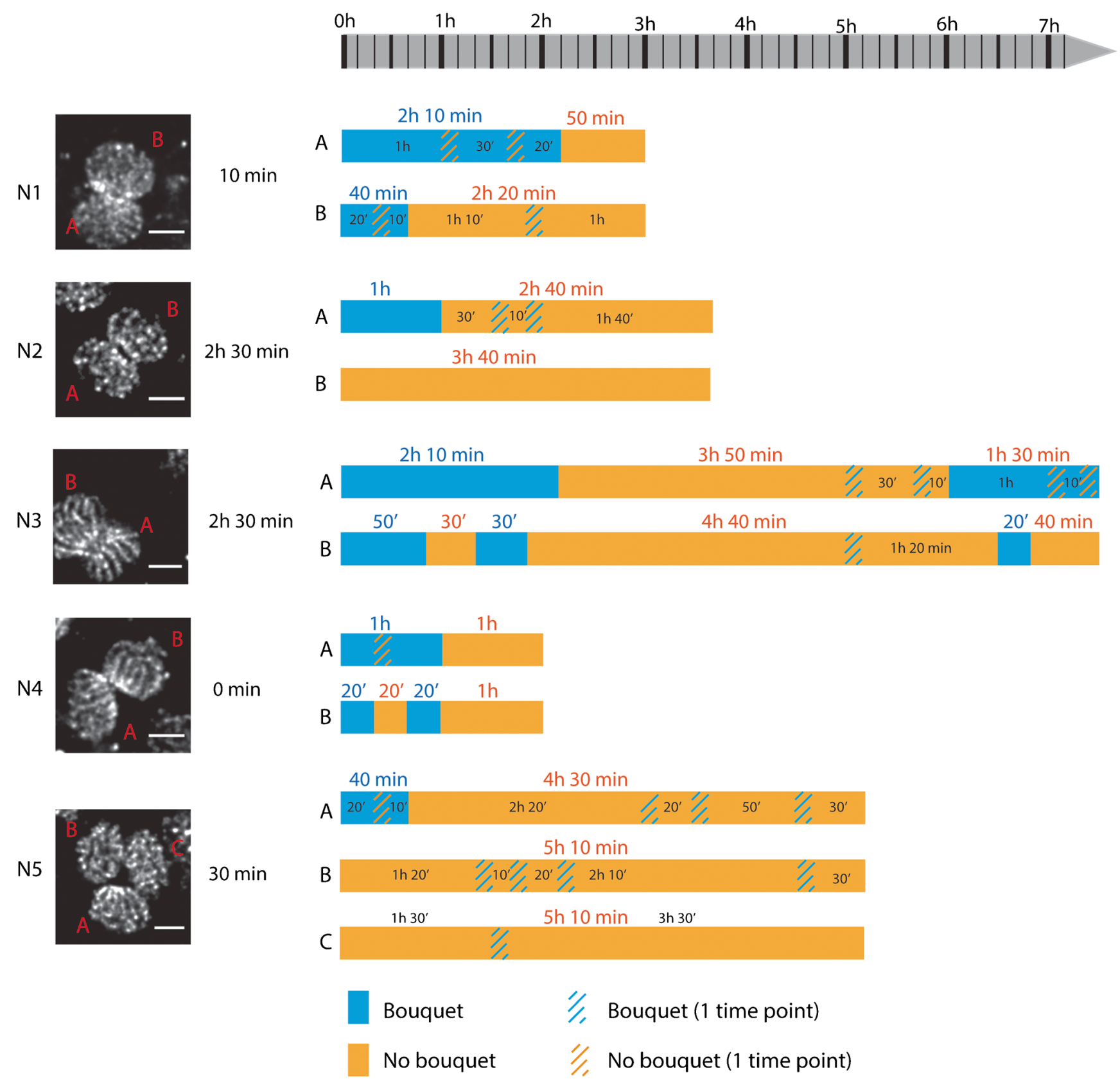

Fig. 6 Bouquet progression in oocytes. Five groups of zygotene nuclei of two different experiments (experiment 1: N1 and N4; experiment 2: N2, $\mathrm{N} 3$ and N5) were analyzed. Nuclei are ordered from early to late zygotene, from top to bottom of the figure. An image at t0 is showed for N1-N4. For N5, t5 was chosen, to be able to show the three nuclei in the same plane. Scale bar $5 \mu \mathrm{m}$. The amount of time passed up to the first

observed bouquet is indicated before each time bar. Blue bars indicate periods during which a bouquet stage was observed; orange bars indicate periods in which no bouquet was observed for longer than $10 \mathrm{~min}$. Orange diagonal lines indicate loss of clear bouquet configuration at only a single time point; blue diagonal bars indicate apparent bouquet organization of chromosomes at only a single time point

given the fact that both proteins are recruited properly to the $\mathrm{SC}$ on the wild-type background. The aberrant chromatin localization pattern of both SCYCP and SYCPC in the absence of endogenous SYCP3 already explains their lack of functionality. However, in addition, the aberrant timing of transgene activation during meiotic prophase, as revealed on the wildtype background (see below), may also contribute to the lack of rescue of the Sycp3 knockout phenotype.

\section{SYCPC and CSYCP can accumulate on the axes of the SC and their expression does not interfere with fertility}

We observed that, on the wild-type background, both CSYCP and SYCPC localize to the axes of the SC, together with endogenous SYCP3, from pachytene onwards in male mice, and from leptotene onwards in embryonic ovaries at E16.5 or 

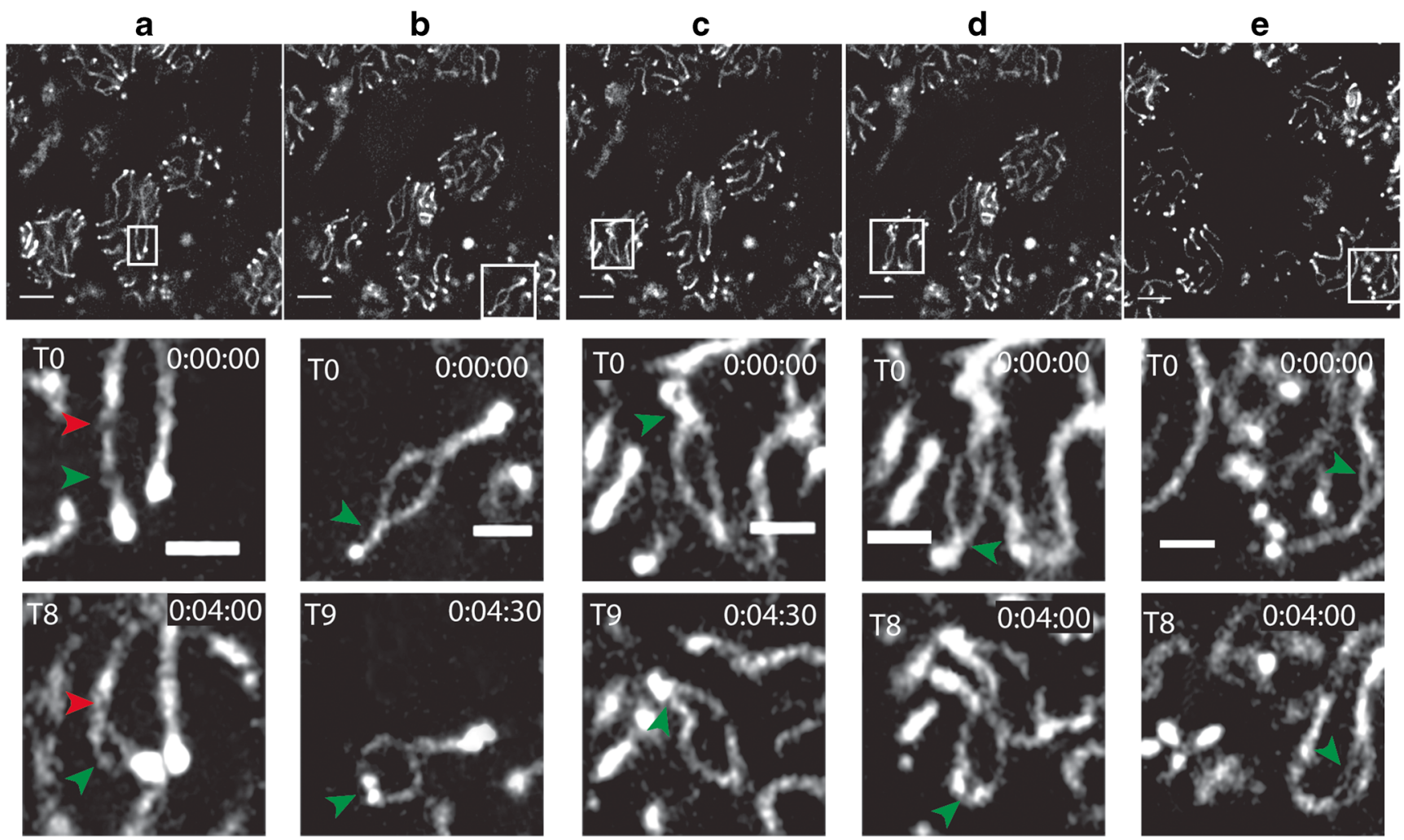

f

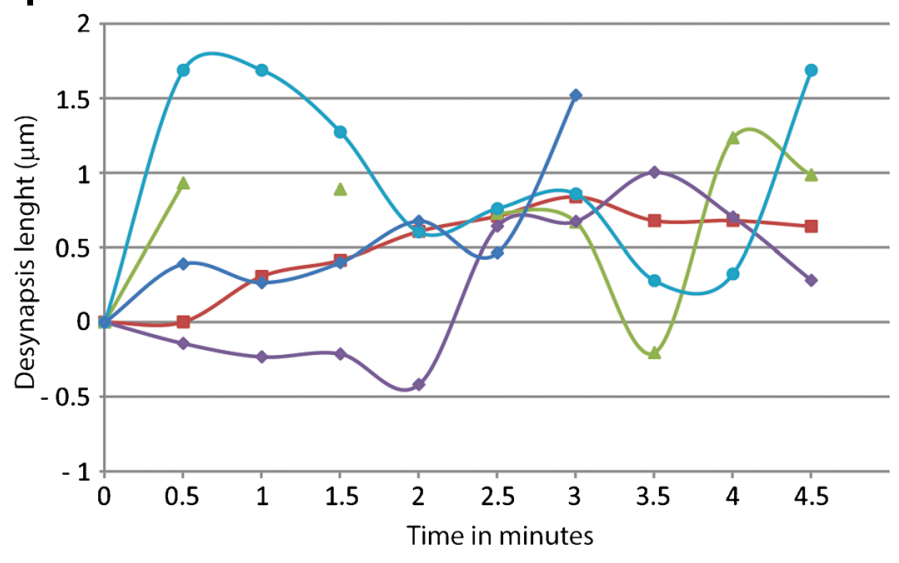

$-a-b \quad r \quad \sim d \rightleftharpoons e$

Fig. 7 Analyses of axis separation in diplotene spermatocytes. a-e Desynapsis progression in five different bivalents of Sycp $3^{+/-}$ CSYCP diplotene spermatocytes. CSYCP is visualized in gray. An overview of the whole field of view at T0 is shown in the upper panels (scale bar $5 \mu \mathrm{m}$ ). Below each image, enlargements of the bivalent that desynapses (indicated by a boxed area) are shown at T0 and at T8 or T9 (4 or $4.5 \mathrm{~min}$ later). Scale bar $2 \mu \mathrm{m}$. f Progression of

later. The lack of SYCPC and CSYCP accumulation on axial elements in wild-type early spermatocytes was unexpected, since the Smclbeta promoter fragment that we used has been reported to drive expression from leptotene onwards (Adelfalk et al. 2009). The expression pattern for both transgenes in our mouse models follows the overall pattern of transcriptional activity that has been reported for male meiotic prophase: a g

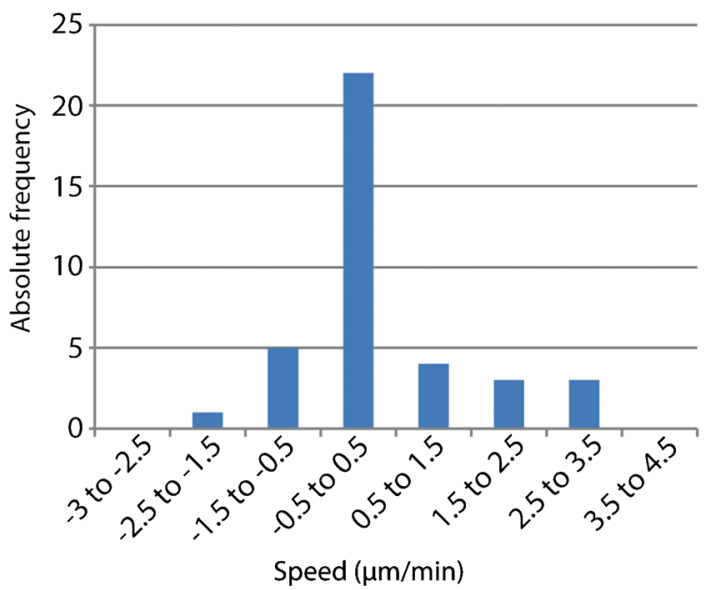

desynapsis in time for each bivalent. Note that bivalent e (light green) could not be measured at $t=1 \mathrm{~min}$ and $t=2 \mathrm{~min}$, because it moved outside the field of view (see video 9). g Frequency distribution of the desynapsis (positive)/resynapsis (negative) speeds of the five bivalents. Speeds were measured as described in the "Materials and methods" section. All speeds of all bivalents were analyzed together

general transcriptional inactivation occurs in early stages of male meiosis (leptotene, zygotene and early pachytene), followed be reactivation from mid pachytene onwards (Kierszenbaum and Tres 1974; Monesi 1964; Page et al. 2012). Thus, it is possible that the transgenes are situated in regions that are subject to this global inactivation mechanism, and therefore can only be transcribed from pachytene onwards 


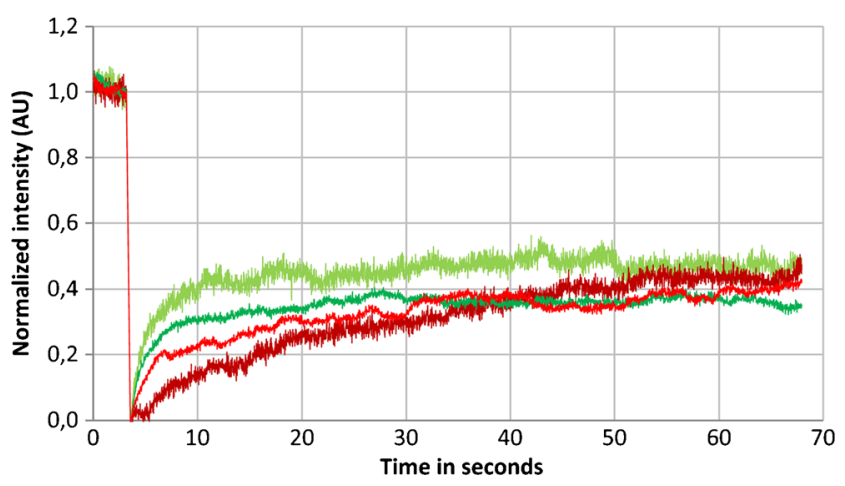

Fig. 8 Fluorescence recovery after photobleaching of SYCPC and CSYCP in autosomes of pachytene spermatocytes. Each line represents the averaged and normalized fluorescent recovery of at least ten spermatocyte nuclei of one mouse testis. The results obtained of two Sycp $3^{+/}$SYCPC (green) and two Sycp $3^{+/}$CSYCP (red) males are plotted against time. Total time recording after bleaching $1 \mathrm{~min}$. Time lapse $0.020 \mathrm{~s}$

in males, when global transcription is reactivated. However, we cannot exclude that the observed expression patterns are inherent to the promoter fragment used. In females, expression of the fusion protein was also observed later than expected, and while endogenous SYCP3 can be detected from E13.5 onwards, the tagged proteins were only expressed from E16.5 onwards, when leptotene oocytes still form. Since the tagged proteins are expressed (and incorporated into the LEs) only from pachytene onwards in males, and FRAP analyses showed a final recovery of $40 \%$ for both SYCPC and CSYCP during pachytene, we can conclude that tagged SYCP3 can be recruited to already synapsed axes and exchange there. These data might suggest that a mixed filament of endogenous and tagged SYCP3 forms, whereby endogenous SYCP3 protein also exchanges. However, we cannot exclude that the tagged proteins are loosely associated and do not exchange with endogenous SYCP3.

Remarkably, both Sycp $3^{+/}$SYCPC and Sycp $3^{+/}$CSYCP spermatocytes presented a stronger mCherry signal on the (largely) unsynapsed XY compared to the synapsed autosomes. Also, the telomeric regions of the chromosomal axes are enriched for the fusion proteins. Using antibody staining, to detect both endogenous and tagged SYCP3, we observed a dimmer signal on the $\mathrm{XY}$ due to the single axes, and no enrichment at the telomeres in mid pachytene, similar to the wild-type pattern. This difference might be explained by increased exchange between endogenous SYCP3 and SYCPC/ CSYCP in these regions, if this type of exchange occurs. In this situation, possible differences in the turnover of tagged SYCP3 and endogenous SYCP3 would be relevant. As pachytene progresses, the relative amount of tagged SYCP3 compared to endogenous SYCP3 may increase, allowing an increase in the amount of tagged protein in regions that somehow are exchanging more SYCP3. In the absence of exchange of tagged SYCP3 with endogenous SYCP3, the data can also be explained by the presence of more binding sites for the fusion protein, in the unsynapsed $\mathrm{XY}$ and telomeric regions, compared to the rest of the SC, in combination with an overall (much) lower expression of the fusion protein compared to endogenous protein, masking this effect in the immunostainings. The extra accumulation of SYCPC and CSYCP on the axial/lateral elements of the XY pair adds another interesting feature to the structural properties the $\mathrm{XY}$ pair, in addition to the described relatively short chromatin loops and long lateral elements of the pseudoautosomal region of the XY pair (Kauppi et al. 2011), which is the part that actually displays stable synapsis.

Wild-type males and females expressing either transgene displayed normal progression through meiotic prophase, and overall fertility was not affected. In addition, we observed no detrimental effects of the long-term and short imaging experiments, since the overall morphology of the nuclei and the SC had not changed, and cellular movements kept occurring within the ovaries and tubule fragments that were cultured for short or long (overnight) periods. In the tubule cultures, contractions of the tubules also still occurred after overnight imaging. Thus, despite the fact that the proteins cannot fully replace endogenous SYCP3, our system provides a tool to study meiosis in living cells, in the natural context of the seminiferous tubule or the embryonic ovary.

\section{Nuclear rotations and chromosome movements}

We have observed two types of movements inside the cultured seminiferous tubules and the ovaries of our transgenic mouse models: the rotation of the nucleus itself and movement of the $\mathrm{SC}$ inside the nucleus. The analysis of the movement of the SC inside the nucleus revealed an average speed of the SC of the XY of $24 \pm 15 \mathrm{~nm} / \mathrm{s}$. Other groups measured telomere velocity in mouse pachytene cells and observed $130 \mathrm{~nm} / \mathrm{s}$ (Shibuya et al. 2014) or $36 \pm 14.8 \mathrm{~nm} / \mathrm{s}$ (Lee et al. 2015). The latter result nicely fits our data; a somewhat faster movement of the telomeres compared to the rest of the chromosome is to be expected, since the movement of the telomeres along the nuclear envelope may displace also the rest of the chromosome, but to a lesser extent. Related to this, in maize pachytene cells, it has also been observed that chromosome ends travel faster than interstitial regions of the same chromosomes (Sheehan and Pawlowski 2009). Whether the chromosome movements that we observed in late meiotic prophase have any function is unknown. However, it seems to be an evolutionary conserved feature, since it has also been described for budding yeast, where telomere speed was similar in paired and non-paired configurations (Scherthan et al. 2007; Conrad et al. 2008; Koszul et al. 2008). For budding yeast, it has been suggested that chromosome movements after chromosome pairing could be required to help to prevent topological entanglements or to eliminate them if they persist up to the 
pachytene stage (Koszul et al. 2008). Alternatively, late telomere movements could have a role in late steps of recombination like Holliday junction formation and resolution in pachytene (Scherthan et al. 2007).

Existence of nuclear rotation was also mentioned in previous studies in rat spermatocytes (Parvinen and Soderstrom 1976), but not further characterized. We have analyzed the rotation of the nuclei in zygotene oocytes and pachytene spermatocytes, and we have observed longer time periods of rotation in a single direction in oocytes in early meiotic prophase, compared to pachytene spermatocytes. This observation is consistent with the fact that rotational movements accompany chromosome pairing, and the idea that perhaps concerted movements of telomeres to the same site on the nuclear envelope induce rotations of the entire nucleus (Scherthan et al. 1996). Indeed, the function of the rotational movements in promoting pairing seems to be conserved in evolution. However, the pattern of these movements appears to vary among organisms. In maize meiocytes, oscillations back and forth are the most common type of nuclear rotational movement, although rotations in a single direction (small and large) have also been observed (Sheehan and Pawlowski 2009). In fission yeast, oscillations of the entire nucleus between the two poles (horsetail movements) start just after bouquet formation. The impairment of these movements results in reduced pairing of the homologous chromosomes (Yamamoto and Hiraoka 2001; Saito et al. 2005; Chacon et al. 2016). Interestingly, horsetail movements continue after pairing has been achieved. Their inhibition after pairing produces mis-segregation of the chromosomes during the first meiotic division. Such late oscillations may prevent prolonged associations of the homologous chromosomes once pairing has been achieved, which may lead to irresolvable recombination intermediates and segregation failure (Chacon et al. 2016). The "wiggling" of late spermatocyte nuclei could assist in a similar process, also in combination with the movement of the chromosomes.

\section{The bouquet stage in mouse oocytes is dynamic}

It is known that primordial germ cells form cysts connected by intercellular bridges in the ovaries of E11.5 to E17.5 mice (Pepling 2006; Pepling and Spradling 1998). Accordingly, we have observed that E16.5 oocytes frequently formed groups of two, and occasionally three, nuclei that localized in a single cytoplasm. Bouquet formation within such syncytia was frequently synchronized and the opposite localization of the telomere clusters gave such nuclei a "kissing" appearance. The intercellular bridges could be involved in this synchronization, since bundles of microtubules have been shown to traverse intercellular bridges (Pepling and Spradling 1998). These microtubules are implicated in cellular transport among cells in a cyst (Pepling and Spradling 1998), but they could also play an important role in the synchronization of bouquet formation and connect the cytoplasmic components of the proteins that mediate the clustering of telomeres in association with the nuclear membrane.

In female mice, bouquet formation has been reported to peak at mid to late zygotene and to persist in a substantial proportion of pachytene oocytes (Tankimanova et al. 2004). In accordance with this observation on fixed samples, the living nuclei in which we observed a bouquet were most often in a mid-late zygotene stage. Because of the limitations of our imaging system, we cannot exclude that we underestimate the duration of the bouquet stage. However, what is clear is that the bouquet stage is highly dynamic. During the recordings, some nuclei exhibited two cycles of telomere clustering, dissolution, and reclustering. In some nuclei within a syncytium, the bouquet of one nucleus dissolved while in the other it remained, followed by reclustering of the telomeres in the nucleus that had previously lost its bouquet configuration. In other syncytia, clustering and dissolution cycles occurred more synchronously and sometimes a dissolution/ reclustering cycle could be very rapid (around $20 \mathrm{~min}$ ). Maybe some events of dissolution occur when not all the chromosomes are synapsed or when (partial) nonhomologous associations are present that activate a feedback mechanism that triggers reformation of the bouquet, to allow complete synapsis of the remaining unsynapsed axes. Alternatively, or in addition, telomere declustering and reclustering events could be important to solve whole chromosome entanglements, known as "interlocks" that are present during synapsis but absent by the end of pachytene (and therefore resolved) (Zickler and Kleckner 2015; Zickler and Kleckner 2016).

\section{Desynapsis}

Finally, we were also able to follow axis separation, representing desynapsis in male diplotene spermatocytes. In these cases, we observed initiation of desynapsis in the interstitial region of the chromosomes and progression of desynapsis towards the telomeres. Since we could only follow five bivalents, we cannot exclude that desynapsis events might also start from the telomeres. The speed of desynapsis varied; we observed discontinuous progression, and even brief events of resynapsis, indicating that desynapsis occurs in bursts and may also halt for a certain time period. Given the overall normal development of spermatocytes even during overnight imaging experiments, and the observation that multiple desynapsis or resynapsis proceedings could be traced for a single bivalent, we infer that our settings do not interfere with overall chromosome behavior during meiotic prophase. If we 
estimate the total length of the SC to be around $200 \mu \mathrm{m}$ (Baarends et al. 2003), it might be expected that desynapsis would not take much longer than $200 \mathrm{~min}$, since multiple bivalents are usually observed to desynapse simultaneously. However, analyses of fixed samples has indicated that in mouse, diplotene lasts around 3 days (Oud et al. 1979). Thus, it can be concluded that the duration of desynapsis is limited by factors other than the actual constraints of the desynapsis process itself.

In summary, we have used the mCherry SYCP3 and SYCP3 mCherry male and female mice to study synaptonemal complex and chromosome dynamics in vivo, for the first time in the natural context of the seminiferous tubule or the ovary, during an imaging time frame of several hours. We have focused on bouquet formation, nuclear rotation, and desynapsis events. In addition to providing new insights in the dynamics of chromosome behavior in wild-type meiocytes, our method can be used as a tool to study alterations in the chromosome dynamics of meiocytes in gonads of infertile mouse models.

\section{Materials and methods}

\section{Ethics statement}

All animal experiments were approved by the local animal experiments committee DEC Consult and animals were maintained under supervision of the Animal Welfare Officer.

\section{Generation of SYCPC and CSYCP mice}

In order to study the dynamics of the SC, we have generated transgenic mice expressing $\mathrm{N}$ - or $\mathrm{C}$ - terminal fusions of SYCP3 with the mCherry protein. To this end, we first cloned the coding region of the mouse Sycp3 gene into the mCherry $\mathrm{C} 1$ or N1 vector. The mouse Sycp3 coding region was amplified using forward primer $5^{\prime}$ CCGCTCGAGTGCTTCGAGGGTGTG3' and reversed primer 5'CGCGGATCCAGACTCATCAGAATAACATG $3^{\prime}$ to generate CSYCP and forward primer CCGCTCGA GTCAGATGCTTCGAGGGTG and reversed primer 5' CGCGGATCCCAGAATAACATGGATTGAAGAG $3^{\prime}$ to generate SYCPC. Both fragments were cut with XhoI and BamH1 and cloned into the multiple cloning site of the $\mathrm{C} 1$ and N1 vector, respectively. Subsequently, we amplified a $295 \mathrm{bp}$ fragment of the $S m c 1 \beta$-promoter previously described to drive expression from leptotene onwards (Adelfalk et al., 2009) using PCR primers SMC1bFor: 5' CCGCTATTAATCACGGCAAG AAAAGCCC 3' and SMC1bRev: 5' CTAGCTAG CGACCGGTGCCTCAGCC $3^{\prime}$ followed by digestion of the fragment with AseI and NheI and cloning into the two vectors containing the SYCPC and CSYCP constructs by replacing the CMV promoter in these plasmids. Finally, the constructs were digested with EciI, purified and injected in the pronuclei of FVB zygotes using standard methods. Transgenic mice were tested for expression of the transgenes in the testes, and the SYCP3 open reading frame was sequenced to exclude point mutations. Mice carrying the transgenes could be bred to homozygosity without affecting overall health. Animals were genotyped using standard DNA isolation (DNA isolated from tail or toe tips) and PCR procedures using the following primer sets: forward primer 5'CACCATCGTGGAACAGTACG $3^{\prime}$ and reverse primer 5'GGGAGGTGTGGGAGGTTTT3' for CSYCP and forward primer 5'GCAAGGGC GAGGAGGATAAC $3^{\prime}$ and reverse primer 5' TTGCCGATTTCGGCCTATTG 3' for SYCPC.

\section{Mice}

Sycp3 knockout mice were described previously (Yuan et al. 2000) and crossed with SYCPC or CSYCP mice. Testes were isolated from immature and adult male mice. To obtain ovaries containing oocytes at different stages of meiotic prophase, Sycp $3^{+/-}$(or occasionally $S y c p 3^{-/}$in case of females) with or without the CSYCP or SYCPC transgene were mated and ovaries were isolated from embryos at E.15.5, 16.5, or E18.5. (the day at which a plug was observed was considered as day 0.5 of embryonic development, E0.5). Meiotic progression in females differs in terms of timing and stage appearance compared to the male. The more or less synchronous development of oocytes in the embryonic ovary allowed us to predict in which stage the majority of the cells would be depending on the embryonic day of development. In this way, the majority of the oocytes will be at leptotene or zygotene at E15.5 and E16.5 and at pachytene or diplotene at E18.5 (Ashley 2004; Dietrich and Mulder 1983).

\section{Western blotting}

Testes were isolated from SYCPC and CSYCP mice of different ages (SYCPC 11, 16, and 19 days old, CSYCP 12,15 , and 22 days old). Total testis protein isolation and Western blotting of $12 \%$ SDS-PAGE gels were performed as described previously (Mulugeta Achame et al. 2010). Rabbit polyclonal anti-SYCP3 (Lammers et al. 1994) was diluted 1:5000. Peroxidase-labeled secondary antibody (Sigma) was used, and antigen-antibody complexes were detected using a chemoluminescence kit (Du Pont/NEN, Bad Homburg, Germany) according to the instructions provided by the manufacturer. 


\section{Immunocytochemistry}

Spread nuclei of spermatocytes and oocytes were prepared from isolated gonads as described by Peters et al. (1997) and stored at $-80 \mathrm{C}$. Thawed slides were washed in PBS $(3 \times$ $10 \mathrm{~min}$ ), and non-specific sites were blocked with $0.5 \% \mathrm{w} / \mathrm{v}$ BSA and $0.5 \% w / v$ milk powder in PBS. Primary antibodies were diluted in $10 \% w / v$ BSA in PBS, and incubations were performed overnight at room temperature in a humid chamber. Subsequently, slides were washed $(3 \times 10 \mathrm{~min})$ in PBS, blocked in $10 \% v / v$ normal goat serum (Sigma) in blocking buffer (supernatant of 5\% w/v milk powder in PBS centrifuged at $14,000 \mathrm{rpm}$ for $10 \mathrm{~min}$ ), and incubated with secondary antibodies in $10 \%$ normal goat serum in blocking buffer at room temperature for $2 \mathrm{~h}$. Finally, slides were washed $(3 \times$ 10 min) in PBS and embedded in Prolong Gold with DAPI (Invitrogen). For stainings involving two primary antibodies generated in the same species, the immunostaining was performed sequentially. After adding one of the two primary antibodies, and its detection on the next day with a secondary antibody, the other primary antibody was added and detected the day thereafter with a secondary antibody of a different color. Note that the protein detected by the antibody added on the first day would be visible in the colors of each of the two secondary antibodies, while the protein detected by the primary antibody that was added on the second day would be visible only in the color of the last secondary antibody.

Primary antibodies: mouse monoclonal anti-SYCP3 (ABCAM:ab97672) at 1:200, rabbit polyclonal anti-SYCP3 (Lammers et al. 1994) at 1:10000, guinea pig polyclonal antiSYCP2 (Yang et al. 2006) at 1:100, rabbit polyclonal antiSYCP2 (Offenberg et al. 1998) at 1:400, rabbit polyclonal anti-RAD51 (Essers et al. 2002) at 1:500, rabbit polyclonal anti-REC8 (N-terminus, affinity purified) at 1:50 (Eijpe et al. 2003), rabbit polyclonal anti-SYCP1 (Meuwissen et al. 1992) at 1:5000, mouse monoclonal anti-MLH1 (cat. 551,091, BD Pharmigen) at 1:25. For secondary antibodies, we used a goat anti-rabbit alexa $488 \mathrm{IgG}$, goat anti-rabbit alexa $633 \mathrm{IgG}$, goat anti-mouse alexa 488, and goat anti-mouse alexa $546 \mathrm{IgG}$, all at 1:500 dilution.

Fluorescent images were obtained using a fluorescence microscope (Axioplan 2; Carl Zeiss) equipped with a digital camera (Coolsnap-Pro; Photometrics). Confocal images were taken using a Zeiss LSM700 confocal, equipped with a digital camera (Axiocam MRm Rev.3 1388X1040 monochrome camera for epi-fluorescence). Foci were counted using ImageJ software (FIJI); we counted foci using the find maxima function and set the noise tolerance manually.

\section{Staging of spermatocytes and oocytes}

Staging of wild-type spermatocytes and oocytes was based on the pattern of the SYCP3 or REC8 $\left(\mathrm{Sycp} 3^{-/-}\right.$ spermatocytes and oocytes). In addition, RAD51 was used in order to distinguish between zygotene (250-100 foci, as the number of foci decreases as synapsis progresses) and diplotene oocytes ( $<10$ foci (Moens et al. 1997). Alternatively, the presence of MLH1 foci was used as marker of pachytene and diplotene oocytes.

\section{Isolation of testis tubules and embryonic ovaries for culture}

Adult mouse testes from transgenic mice carrying the CSYCP or SYCPC transgene were isolated and dissected following a previously described protocol (van der Laan et al. 2004) with some modifications. Decapsulated testes were immersed in $20 \mathrm{ml}$ Dulbecco's phosphate-buffered saline (Invitrogen, Carlsbad, CA, USA) containing $1.1 \mathrm{mM} \mathrm{Ca}^{2+}$, $0.52 \mathrm{mM} \mathrm{Mg} 2^{+}, 6 \mathrm{mM}$ DL-lactic acid, and $5.6 \mathrm{mM}$ glucose $\left(\mathrm{PBS}^{+}\right)$, in the presence of collagenase $(1 \mu \mathrm{g} / \mu \mathrm{l}$, type 1 Worthington) and hyaluronidase $(0.5 \mu \mathrm{g} / \mu \mathrm{l}, \mathrm{H} 3884$ Sigma $)$ in a $50-\mathrm{ml}$ falcon tube. The testes were then incubated in a water bath at $33^{\circ} \mathrm{C}$ and shaken at 90 cycles/min, amplitude of $20 \mathrm{~mm}$, for $5 \mathrm{~min}$. The incubation was stopped when the enzymatic digestion had resulted in dissociation of the interstitial tissue and disengagement of testis tubules. Tubules were separated from interstitial cells by washing in $\mathrm{PBS}^{+}$twice. Next, tubules were placed in a 30-mm BSA-coated Petri dish, where the tubules were separated from each other using dissection tweezers (Nr. 5). After cutting small fragments, these were carefully transferred to a 15-mm BSA-coated Petri dish. After collecting a pool of fragments, we used dissection tweezers (Nr. 5) to place 4-5 of these fragments into a 50- $\mu$ l drop containing 1:4 v/v (RPMI + 10\% KRS culture medium)/ Cultrex ${ }^{\circledR}$ Basement Membrane Extract (BME), type 2; Trevigen pipetted onto a 24-mm laminin-coated cover slip in each live cell chamber. The chambers with the tubules were centrifuged $5 \mathrm{~min}$ at $500 \mathrm{rpm}$ and $4{ }^{\circ} \mathrm{C}$. Next, the chambers were incubated for $30 \mathrm{~min}$ at $33^{\circ} \mathrm{C}$ to allow the BME to jellify. Finally, $2 \mathrm{ml}$ of RPMI $+10 \%$ KRS culture medium was added. For embryonic ovary culture, pregnant females were killed at different time points after timed matings. Embryos were collected and ovaries were isolated and placed temporarily in $\mathrm{PBS}^{+}$(without DL-lactic acid). Once all the ovaries had been collected, they were transferred to the live cell chamber, prepared as described for the tubules. The rest of the protocol was also identical, with the exception of the medium ( $\alpha \mathrm{MEM}$, Gibco) and the temperature $\left(37^{\circ} \mathrm{C}\right.$ instead of 33$)$.

\section{Confocal and time-lapse microscopy}

For the time-lapse imaging, we used a Leica SP5 confocal microscope. To specifically follow desynapsis in diplotene spermatocytes, we used a Zeiss LSM880 microscope with airy-scan detector. Chamber and objective $(40 \times 1.25$ 
NA, oil immersion) were kept at $33{ }^{\circ} \mathrm{C}$ for seminiferous tubules and $37{ }^{\circ} \mathrm{C}$ for ovaries. The chambers with the tubules or ovaries were also maintained at $5 \% \mathrm{CO}_{2}$. Red fluorescent images were obtained after excitation with a 594-nm laser and detection through a $600-680$ band pass filter.

For the FRAP analysis, we bleached a single strip of 16 pixels high (pixel size $0.11 \mu \mathrm{m} X Y$ ) that spanned the middle part of the nucleus, for five iterations at high laser intensity (100\% of the 594 and $561 \mathrm{~nm}$ laser). The recovery of fluorescence in the strips was monitored during $1 \mathrm{~min}$ at intervals of $0.020 \mathrm{~s}$ at $6-12 \%$ of the laser intensity applied for bleaching (12\% for the mice carrying the SYCPC transgene and $6 \%$ for the mouse carrying the CSYCP transgene, due to its higher signal intensity). Samples of two mice, one carrying CSYCP and the other carrying SYCPC, were processed together and analyzed the same day. Another set of two mice carrying each variant of the transgene was used to confirm the results.

For the overnight time-lapse experiments, we selected 510 different positions along each tubule or ovary, and for each position, a stack of approximately 30 slices of $1 \mu \mathrm{m}$ was made. The lapse between images was $10 \mathrm{~min}$. For the short videos time-lapse experiments, total time and lapse varied between experiments.

\section{Analysis of the rotation, chromosome movements, and axis separation}

Time-lapse videos of one plane per time point and a time lapse of 1.5-2 s were recorded for this analysis. For the rotation in $\mathrm{X}-\mathrm{Y}$ analysis, single oocytes or spermatocytes were selected. The rotation angle of the time point $t+1$ with respect to $t$ was calculated. For this, the nucleus at $t+1$ was translated $(\mathrm{X}, \mathrm{Y})$ and rotated until the correlation between $t$ and $t+1$ was the highest. From the rotation angle, we calculated the rotation speeds (degrees/s).

To measure the speed of the XY chromosome in pachytene spermatocytes, we measured the distance from the center of the $\mathrm{XY}$ at time $t+1$ to the center at time $t$ and divided this value by the time in seconds.

To measure the speed of desynapsis, the length of the remaining synapsed (bivalent b, d) or desynapsed (bivalent a,c, e) fragment was measured at each analyzed time point, in one single XY plane. The length difference between the two time points was measured and used to calculate the speed. Speeds were determined for each 30-s interval during the time period for which the bivalents could be traced.

Acknowledgments We would like to thank the help of Alex Maas and John-Kong-a-San (Department of Cell Biology, Erasmus MC, Rotterdam, The Netherlands), Christer Höög (Department of Cell and Molecular Biology, Karolinska Institutet, Stockholm, Sweden), and
Rolf Jessberger (Institute of Physiological Chemistry, Medical Faculty Carl Gustav Carus, Technische Universität Dresden, Dresden, Germany).

Funding This work was supported by the Netherlands Organization for Scientific Research (NWO) through ALW Open Program 819.02.020 and through $C W E C H O 104126$ and by the European Commission through EU-FP7-PEOPLE-2011-ITN289880. The funders had no role in the study design, data collection and analysis, decision to publish, or preparation of the manuscript.

\section{Compliance with ethical standards}

Conflict of interest The authors declare that they have no conflict of interest.

Ethical approval All applicable international, national, and/or institutional guidelines for the care and use of animals were followed. All procedures performed in studies involving animals were in accordance with the ethical standards of the institution or practice at which the studies were conducted.

Open Access This article is distributed under the terms of the Creative Commons Attribution 4.0 International License (http:// creativecommons.org/licenses/by/4.0/), which permits unrestricted use, distribution, and reproduction in any medium, provided you give appropriate credit to the original author(s) and the source, provide a link to the Creative Commons license, and indicate if changes were made.

\section{References}

Adelfalk C, Janschek J, Revenkova E, Blei C, Liebe B, Göb E, Alsheimer M, Benavente R, de Boer E, Novak I, Höög C, Scherthan H, Jessberger R (2009) Cohesin SMC1beta protects telomeres in meiocytes. J Cell Biol 187:185-199. https://doi.org/10.1083/jcb. 200808016

Alsheimer M, Baier A, Schramm S, Schutz W, Benavente R (2010) Synaptonemal complex protein SYCP3 exists in two isoforms showing different conservation in mammalian evolution. Cytogenet Genome Res 128:162-168. https://doi.org/10.1159/ 000303341

Ashley T (2004) The mouse "tool box" for meiotic studies. Cytogenet Genome Res 105:166-171. https://doi.org/10.1159/000078186

Baarends WM, Wassenaar E, Hoogerbrugge JW, van Cappellen G, Roest HP, Vreeburg J, Ooms M, Hoeijmakers JHJ, Grootegoed JA (2003) Loss of HR6B ubiquitin-conjugating activity results in damaged synaptonemal complex structure and increased crossing-over frequency during the male meiotic prophase. Mol Cell Biol 23:11511162

Baudat F, Manova K, Yuen JP, Jasin M, Keeney S (2000) Chromosome synapsis defects and sexually dimorphic meiotic progression in mice lacking spo11. Mol Cell 6:989-998

Boateng KA, Bellani MA, Gregoretti IV, Pratto F, Camerini-Otero RD (2013) Homologous pairing preceding SPO11-mediated doublestrand breaks in mice. Dev Cell 24:196-205. https://doi.org/10. 1016/j.devcel.2012.12.002

Chacon MR, Delivani P, Tolic IM (2016) Meiotic nuclear oscillations are necessary to avoid excessive chromosome associations. Cell Rep 17:1632-1645. https://doi.org/10.1016/j.celrep.2016.10.014

Conrad MN, Lee CY, Chao G, Shinohara M, Kosaka H, Shinohara A, Conchello JA, Dresser ME (2008) Rapid telomere movement in 
meiotic prophase is promoted by NDJ1, MPS3, and CSM4 and is modulated by recombination. Cell 133:1175-1187

Costa $Y$ et al (2005) Two novel proteins recruited by synaptonemal complex protein 1 (SYCP1) are at the centre of meiosis. J Cell Sci 118: 2755-2762. https://doi.org/10.1242/jcs.02402

Dietrich AJ, Mulder RJ (1983) A light- and electron microscopic analysis of meiotic prophase in female mice. Chromosoma 88: 377-385

Dobson MJ, Pearlman RE, Karaiskakis A, Spyropoulos B, Moens PB (1994) Synaptonemal complex proteins: occurrence, epitope mapping and chromosome disjunction. J Cell Sci 107(Pt 10):2749-2760

Eijpe M, Offenberg H, Jessberger R, Revenkova E, Heyting C (2003) Meiotic cohesin REC8 marks the axial elements of rat synaptonemal complexes before cohesins SMC1beta and SMC3. J Cell Biol 160: 657-670. https://doi.org/10.1083/jcb.200212080

Essers J, Hendriks RW, Wesoly J, Beerens CEMT, Smit B, Hoeijmakers JHJ, Wyman C, Dronkert MLG, Kanaar R (2002) Analysis of mouse Rad54 expression and its implications for homologous recombination. DNA Repair (Amst) 1:779-793

Hamer G, Gell K, Kouznetsova A, Novak I, Benavente R, Hoog C (2006) Characterization of a novel meiosis-specific protein within the central element of the synaptonemal complex. J Cell Sci 119:40254032. https://doi.org/10.1242/jcs.03182

Hamer G, Novak I, Kouznetsova A, Hoog C (2008) Disruption of pairing and synapsis of chromosomes causes stage-specific apoptosis of male meiotic cells. Theriogenology 69:333-339. https://doi.org/10. 1016/j.theriogenology.2007.09.029

Heyting C (1996) Synaptonemal complexes: structure and function. Curr Opin Cell Biol 8:389-396

Kauppi L, Barchi M, Baudat F, Romanienko PJ, Keeney S, Jasin M (2011) Distinct properties of the XY pseudoautosomal region crucial for male meiosis. Science 331:916-920. https://doi.org/10.1126/ science. 1195774

Kierszenbaum AL, Tres LL (1974) Nucleolar and perichromosomal RNA synthesis during meiotic prophase in the mouse testis. J Cell Biol 60: $39-53$

Kolas NK, Cohen PE (2004) Novel and diverse functions of the DNA mismatch repair family in mammalian meiosis and recombination. Cytogenet Genome Res 107:216-231. https://doi.org/10.1159/ 000080600

Koszul R, Kim KP, Prentiss M, Kleckner N, Kameoka S (2008) Actinmediated motion of meiotic chromosomes. Cell 133:1188-1201. https://doi.org/10.1016/j.cell.2008.04.050

Lammers JH, Offenberg HH, van Aalderen M, Vink AC, Dietrich AJ, Heyting C (1994) The gene encoding a major component of the lateral elements of synaptonemal complexes of the rat is related to X-linked lymphocyte-regulated genes. Mol Cell Biol 14:1137-1146

Lee CY, Conrad MN, Dresser ME (2012) Meiotic chromosome pairing is promoted by telomere-led chromosome movements independent of bouquet formation. PLoS Genet 8:e1002730. https://doi.org/10. 1371/journal.pgen.1002730

Lee CY, Horn HF, Stewart CL, Burke B, Bolcun-Filas E, Schimenti JC, Dresser ME, Pezza RJ (2015) Mechanism and regulation of rapid telomere prophase movements in mouse meiotic chromosomes. Cell Rep 11:551-563. https://doi.org/10.1016/j.celrep.2015.03.045

Lee J, Iwai T, Yokota T, Yamashita M (2003) Temporally and spatially selective loss of Rec8 protein from meiotic chromosomes during mammalian meiosis. J Cell Sci 116:2781-2790. https://doi.org/10. $1242 /$ jcs. 00495

Meuwissen RL, Offenberg HH, Dietrich AJ, Riesewijk A, van Iersel M, Heyting C (1992) A coiled-coil related protein specific for synapsed regions of meiotic prophase chromosomes. EMBO J 11:5091-5100

Moens PB, Kolas NK, Tarsounas M, Marcon E, Cohen PE, Spyropoulos B (2002) The time course and chromosomal localization of recombination-related proteins at meiosis in the mouse are compatible with models that can resolve the early DNA-DNA interactions without reciprocal recombination. J Cell Sci 115:1611-1622

Moens PB, Chen DJ, Shen Z, Kolas N, Tarsounas M, Heng HH, Spyropoulos B (1997) Rad51 immunocytology in rat and mouse spermatocytes and oocytes. Chromosoma 106:207-215

Monesi V (1964) Ribonucleic acid synthesis during mitosis and meiosis in the mouse testis. J Cell Biol 22:521-532

Morelli MA, Werling U, Edelmann W, Roberson MS, Cohen PE (2008) Analysis of meiotic prophase I in live mouse spermatocytes. Chromosom Res 16:743-760. https://doi.org/10.1007/s10577-0081224-8

Mulugeta Achame E, Wassenaar E, Hoogerbrugge JW, Sleddens-Linkels E, Ooms M, Sun ZW, van IJcken WFJ, Grootegoed JA, Baarends WM (2010) The ubiquitin-conjugating enzyme HR6B is required for maintenance of $\mathrm{X}$ chromosome silencing in mouse spermatocytes and spermatids. BMC Genomics 11:367

Offenberg HH, Schalk JA, Meuwissen RL, van Aalderen M, Kester HA, Dietrich AJ, Heyting C (1998) SCP2: a major protein component of the axial elements of synaptonemal complexes of the rat. Nucleic Acids Res 26:2572-2579

Oud JL, de Jong JH, de Rooij DG (1979) A sequential analysis of meiosis in the male mouse using a restricted spermatocyte population obtained by a hydroxyurea/triaziquone treatment. Chromosoma 71 : 237-248

Page J, de la Fuente R, Manterola M, Parra MT, Viera A, Berríos S, Fernández-Donoso R, Rufas JS (2012) Inactivation or non-reactivation: what accounts better for the silence of sex chromosomes during mammalian male meiosis? Chromosoma 121:307-326. https://doi. org/10.1007/s00412-012-0364-y

Page SL, Hawley RS (2004) The genetics and molecular biology of the synaptonemal complex. Annu Rev Cell Dev Biol 20:525-558. https://doi.org/10.1146/annurev.cellbio.19.111301.155141

Parvinen M, Soderstrom KO (1976) Chromosome rotation and formation of synapsis. Nature 260:534-535

Pelttari J, Hoja MR, Yuan L, Liu JG, Brundell E, Moens P, SantucciDarmanin S, Jessberger R, Barbero JL, Heyting C, Hoog C (2001) A meiotic chromosomal core consisting of cohesin complex proteins recruits DNA recombination proteins and promotes synapsis in the absence of an axial element in mammalian meiotic cells. Mol Cell Biol 21:5667-5677. https://doi.org/10.1128/MCB.21.16.56675677.2001

Pepling ME (2006) From primordial germ cell to primordial follicle: mammalian female germ cell development. Genesis 44:622-632. https://doi.org/10.1002/dvg.20258

Pepling ME, Spradling AC (1998) Female mouse germ cells form synchronously dividing cysts. Development 125:3323-3328

Peters AH, Plug AW, van Vugt MJ, de Boer P (1997) A drying-down technique for the spreading of mammalian meiocytes from the male and female germline. Chromosome Res 5:66-68

Roest HP, van Klaveren J, de Wit J, van Gurp CG, Koken MHM, Vermey M, van Roijen JH, Hoogerbrugge JW, Vreeburg JTM, Baarends WM, Bootsma D, Grootegoed JA, Hoeijmakers JHJ (1996) Inactivation of the HR6B ubiquitin-conjugating DNA repair enzyme in mice causes male sterility associated with chromatin modification. Cell 86:799-810

Revenkova E, Eijpe M, Heyting C, Gross B, Jessberger R (2001) Novel meiosis-specific isoform of mammalian SMC1. Mol Cell Biol 21: 6984-6998

Robert T, Nore A, Brun C, Maffre C, Crimi B, Bourbon HM, de Massy B (2016) The TopoVIB-like protein family is required for meiotic DNA double-strand break formation. Science 351:943-949. https://doi.org/10.1126/science.aad5309

Rog O, Dernburg AF (2015) Direct visualization reveals kinetics of meiotic chromosome synapsis. Cell Rep. https://doi.org/10.1016/j. celrep.2015.02.032 
Romanienko PJ, Camerini-Otero RD (2000) The mouse spo11 gene is required for meiotic chromosome synapsis. Mol Cell 6:975-987

Royo H, Polikiewicz G, Mahadevaiah SK, Prosser H, Mitchell M, Bradley A, de Rooij DG, Burgoyne PS, Turner JMA (2010) Evidence that meiotic sex chromosome inactivation is essential for male fertility. Curr Biol 20:2117-2123. https://doi.org/10.1016/j. cub.2010.11.010

Saito TT, Tougan T, Okuzaki D, Kasama T, Nojima H (2005) Mcp6, a meiosis-specific coiled-coil protein of Schizosaccharomyces pombe, localizes to the spindle pole body and is required for horsetail movement and recombination. J Cell Sci 118:447-459. https:// doi.org/10.1242/jcs.01629

Salonen K, Paranko J, Parvinen M (1982) A colcemid-sensitive mechanism involved in regulation of chromosome movements during meiotic pairing. Chromosoma 85:611-618

Scherthan H, Wang H, Adelfalk C, White EJ, Cowan C, Cande WZ, Kaback DB (2007) Chromosome mobility during meiotic prophase in Saccharomyces cerevisiae. Proc Natl Acad Sci U S A 104:16934 16939

Scherthan H (2007) Telomere attachment and clustering during meiosis. Cell Mol Life Sci 64:117-124. https://doi.org/10.1007/s00018-0066463-2

Scherthan H, Weich S, Schwegler H, Heyting C, Harle M, Cremer T (1996) Centromere and telomere movements during early meiotic prophase of mouse and man are associated with the onset of chromosome pairing. J Cell Biol 134:1109-1125

Schramm S, Fraune J, Naumann R, Hernandez-Hernandez A, Höög C, Cooke HJ, Alsheimer M, Benavente R (2011) A novel mouse synaptonemal complex protein is essential for loading of central element proteins, recombination, and fertility. PLoS Genet 7: e1002088. https://doi.org/10.1371/journal.pgen.1002088

Sheehan MJ, Pawlowski WP (2009) Live imaging of rapid chromosome movements in meiotic prophase I in maize. Proc Natl Acad Sci U S A 106:20989-20994. https://doi.org/10.1073/pnas.0906498106

Shibuya H, Morimoto A, Watanabe Y (2014) The dissection of meiotic chromosome movement in mice using an in vivo electroporation technique. PLoS Genet 10:e1004821. https://doi.org/10.1371/ journal.pgen.1004821
Stewart CL, Burke B (2014) The missing LINC: a mammalian KASHdomain protein coupling meiotic chromosomes to the cytoskeleton. Nucleus 5:3-10. https://doi.org/10.4161/nucl.27819

Syrjanen JL, Pellegrini L, Davies OR (2014) A molecular model for the role of SYCP3 in meiotic chromosome organisation. elife 3. https:// doi.org/10.7554/eLife. 02963

Tankimanova M, Hulten MA, Tease C (2004) The initiation of homologous chromosome synapsis in mouse fetal oocytes is not directly driven by centromere and telomere clustering in the bouquet. Cytogenet Genome Res 105:172-181. https://doi.org/10.1159/ 000078187

van der Laan R et al (2004) Ubiquitin ligase Rad18Sc localizes to the XY body and to other chromosomal regions that are unpaired and transcriptionally silenced during male meiotic prophase. J Cell Sci 117: 5023-5033. https://doi.org/10.1242/jcs.01368

Wang H, Hoog C (2006) Structural damage to meiotic chromosomes impairs DNA recombination and checkpoint control in mammalian oocytes. J Cell Biol 173:485-495. https://doi.org/10.1083/jcb. 200512077

Yamamoto A, Hiraoka Y (2001) How do meiotic chromosomes meet their homologous partners?: lessons from fission yeast. BioEssays 23:526-533. https://doi.org/10.1002/bies.1072

Yang F, De La Fuente R, Leu NA, Baumann C, McLaughlin KJ, Wang PJ (2006) Mouse SYCP2 is required for synaptonemal complex assembly and chromosomal synapsis during male meiosis. J Cell Biol 173: 497-507. https://doi.org/10.1083/jcb.200603063

Yuan L, Liu JG, Hoja MR, Wilbertz J, Nordqvist K, Hoog C (2002) Female germ cell aneuploidy and embryo death in mice lacking the meiosis-specific protein SCP3. Science 296:1115-1118. https:// doi.org/10.1126/science.1070594

Yuan L, Liu JG, Zhao J, Brundell E, Daneholt B, Hoog C (2000) The murine SCP3 gene is required for synaptonemal complex assembly, chromosome synapsis, and male fertility. Mol Cell 5:73-83

Zickler D, Kleckner N (2015) Recombination, pairing, and synapsis of homologs during meiosis. Cold Spring Harb Perspect Biol 7. https:// doi.org/10.1101/cshperspect.a016626

Zickler D, Kleckner N (2016) A few of our favorite things: pairing, the bouquet, crossover interference and evolution of meiosis. Semin Cell Dev Biol 54:135-148. https://doi.org/10.1016/j.semcdb.2016. 02.024 